\title{
WEIGHTED ERROR ESTIMATES OF THE CONTINUOUS INTERIOR PENALTY METHOD FOR SINGULARLY PERTURBED PROBLEMS
}

\author{
ERIK BURMAN, JOHNNY GUZMÁN, AND DMITRIY LEYKEKHMAN
}

\begin{abstract}
In this paper we analyze local properties of the Continuous Interior Penalty (CIP) Method for a model convection-dominated singularly perturbed convection-diffusion problem. We show weighted a priori error estimates, where the weight function exponentially decays outside the subdomain of interest. This result shows that locally, the CIP method is comparable to the Streamline Diffusion (SD) or the Discontinuous Galerkin (DG) methods.
\end{abstract}

\section{INTRODUCTION}

The Continuous Interior Penalty (CIP) method was originally proposed by Douglas and Dupont [9] for parabolic and elliptic equations. The idea was to add a penalization term on the gradient jumps in order to increase robustness for elliptic problems with a dominating convection term. The case of optimal convergence in the high Péclet number regime was analyzed by Burman and Hansbo [2] and Burman [3] for first order conforming and non-conforming approximation and in the framework of $h p$-finite elements by Burman and Ern [5].

In this paper, we are interested in approximating the solution $u$ of the following model problem

$$
\begin{array}{rlrl}
-\varepsilon \Delta u+u_{x}+u & =f & & \text { in } \Omega, \\
u=0 & & \text { on } \partial \Omega,
\end{array}
$$

where $\Omega$ is a polygonal domain, $0<\varepsilon \ll 1$, and $f \in L^{2}(\Omega)$.

Let $U$ denote the approximate solution and $h$ the quality of the mesh. Typically the error is shown to satisfy

$$
\|u-U\|_{L^{2}(\Omega)} \leq C h^{k+\frac{1}{2}}\|u\|_{H^{k+1}(\Omega)}
$$

in the high Péclet number regime, where $k$ is the polynomial order and assuming $u$ has sufficient regularity. Optimal convergence in $h$ of the error in the streamline derivative can also be derived. These results are similar to the typical estimates for other stabilized methods such as Discontinuous Galerkin (DG) method or the Streamline-Diffusion (SD) method [13].

The right-hand side of these estimates, however, depends on a global Sobolev norm. This norm may be large in the presence of layers. Therefore the estimates can be considered to be of practical interest only in case the solution is smooth.

Date: Feb 07, 2007.

1991 Mathematics Subject Classification. 65N30,65N15.

Key words and phrases. singularly perturbed, convection-diffusion, local error analysis, continuous interior penalty method. 
However, many problems of interest do not have smooth solutions. Very often they exhibit "nonsmooth behavior", like shocks, boundary or interior layers and interface discontinuities. Model problem (1.1) for example is known to exhibit internal parabolic layer of order $O(\sqrt{\varepsilon} \log 1 / \varepsilon)$ and exponential outflow layers of order $O(\varepsilon \log 1 / \varepsilon)$.

In designing a numerical method it is important to know how the method will behave in the neighborhood of such a discontinuity, and whether or not the resulting effects are global or local. For example when under-resolved layers are present it is well known that the standard Galerkin method suffers from oscillations that pollute the whole solution.

One approach to assess the propagation of perturbations for a given method is to prove that the local error away from the (possibly under-resolved) layer has optimal convergence. An appealing way to prove such results is by using weighted $a$ priori error estimates to prove local error estimates as was done for the StreamlineDiffusion method by Johnson, Nävert and Pitkäranta in the case of local $L^{2}$-norm estimates [13]. The analysis was then extended by Johnson, Wahlbin and Schatz to $L^{\infty}$-norm estimates [14]. Recently, similar results were proved in the case of the residual free bubble method by Sangalli [16] and the Discontinuous Galerkin method by Guzmán [12].

The CIP method is one instance of a class of symmetric stabilization methods that has received increasing interest lately. Examples of other members of this group are the subgrid viscosity method proposed by Guermond [11], the orthogonal subscale stabilization proposed by Codina and Blasco [8], and the local projection stabilization proposed by Becker and Braack [1].

In this work we prove weighted a priori error estimates for the CIP method. To our knowledge this is the first time such estimates have been proved for a method from the class of symmetric stabilizations. We show that the CIP method has the same upwind and crosswind error propagation properties as the SD-method. In particular the penalization of the jumps of the crosswind derivative allows an improved estimate of the error in the crosswind derivative in the case of piecewise linear approximation.

Compared to the DG-method or the SD-method the proof of local estimates for the CIP-method is more involved. This is due to the fact that the stabilization only controls the part of the streamline derivative that is not in the finite element space. Therefore the desired control of the streamline derivative is obtained in a more implicit fashion than for the other methods.

In order to avoid unnecessary technicalities we consider the simple model problem (1.1) with constant convection velocity and reaction terms. Also, for the sake of simplicity, we only give the detailed proof for the case of first order polynomial approximation. However the present analysis extends to the more general case of high polynomial order and also allows to prove $L^{\infty}$-norm error estimates. We will comment on this in the final section of the paper. In the remaining part of this section we will introduce the CIP-method for problem (1.1) and we state the main results.

1.1. The CIP method. Let $\left\{\mathcal{T}_{h}\right\}$ be a one-parameter family of face-to-face triangulations of $\Omega$, with $h=\sup _{T \in \mathcal{T}_{h}} h_{T}$, where $h_{T}=\operatorname{diam}(T)$. The triangulations are assumed to be globally quasi-uniform, i.e. (if necessary after a renormalization 
of $h)$,

$$
\operatorname{diam} T \leq h \leq C(\text { meas } T)^{1 / 2} \quad \forall T \in \mathcal{T}_{h} .
$$

Through out this paper we assume $0<h<1$. By $V_{h}$ we will denote a finite dimensional space of continuous piecewise linear polynomial functions. We will not pose any boundary conditions on $V_{h}$. We let $\mathcal{E}_{h}^{\partial}$ be the collection of boundary edges, $\mathcal{E}_{h}^{0}$ be the collection of interior edges corresponding to $\mathcal{T}_{h}$, and $\mathcal{E}_{h}=\mathcal{E}_{h}^{0} \cup \mathcal{E}_{h}^{\partial}$. The Continuous Interior Penalty approximation $U \in V_{h}$ is defined as the unique solution to

$$
B(U, v)=\int_{\Omega} f v, \quad \forall v \in V_{h}
$$

where

$$
B(u, v)=\varepsilon A(u, v)+M(u, v)+J_{\|}(u, v)+J_{\perp}(u, v),
$$

with

$$
\begin{aligned}
A(u, v) & =\int_{\Omega} \nabla u \cdot \nabla v-\int_{\partial \Omega}\left(\frac{\partial u}{\partial n} v+\frac{\partial v}{\partial n} u\right) d s+\frac{\gamma_{b c}}{h} \int_{\partial \Omega} u v d s \\
M(u, v) & =\int_{\Omega} u_{x} v+\int_{\Omega} u v+\int_{\partial \Omega^{-}} u v\left|n_{x}\right| d s \\
J_{\|}(u, v) & =h^{2} \sum_{e \in \mathcal{E}_{h}^{0}} \int_{e}\left[u_{x}\right]\left[v_{x}\right] d s \\
J_{\perp}(u, v) & =h^{2} \sum_{e \in \mathcal{E}_{h}^{0}} \int_{e}\left[u_{y}\right]\left[v_{y}\right] d s+h^{1 / 2} \int_{\partial \Omega} u v\left|n_{y}\right|^{2} d s .
\end{aligned}
$$

Here $n=\left(n_{x}, n_{y}\right)$ is the outward unit vector to $\partial \Omega$. The inflow part of the boundary $\partial \Omega^{-} \subset \partial \Omega$ is given by

$$
\partial \Omega^{-}=\left\{(x, y) \in \partial \Omega: n_{x}(x, y)<0\right\} .
$$

The constant $\gamma_{b c}$ is a boundary penalty parameter that has to be chosen large enough in order to guarantee stability.

The choice of $h^{2}$ in the definition of the stability terms $J(u, v)$ is not essential and can be replaced with other expressions (cf. [3]). The jump operator is given by

$$
[v](x, y)=\lim _{t \rightarrow 0^{+}}\left(v\left((x, y)-t n_{e}\right)-v\left((x, y)+t n_{e}\right)\right),
$$

where $e$ is a mesh interior edge, $(x, y) \in e$, and $n_{e}$ is a fixed unit vector normal to the edge $e$. The last term of $J_{\perp}$ does not appear in the original definition of the CIP method appearing in [3]. We added this term in order to improve the width of the crosswind layer to order $\max \left(\varepsilon^{1 / 2}, h^{3 / 4}\right) \log (1 / h)$. Without this term we can only prove that the width of the crosswind layer is of order $h^{1 / 2} \log (1 / h)$.

By the regularity theory $u \in H^{\frac{3}{2}+\delta}(\Omega)$ for some $\delta>0$ (cf. [10]). Hence $u$ satisfies (1.2) and we have the usual Galerkin orthogonality property

$$
B(u-U, v)=0, \quad \forall v \in V_{h} .
$$




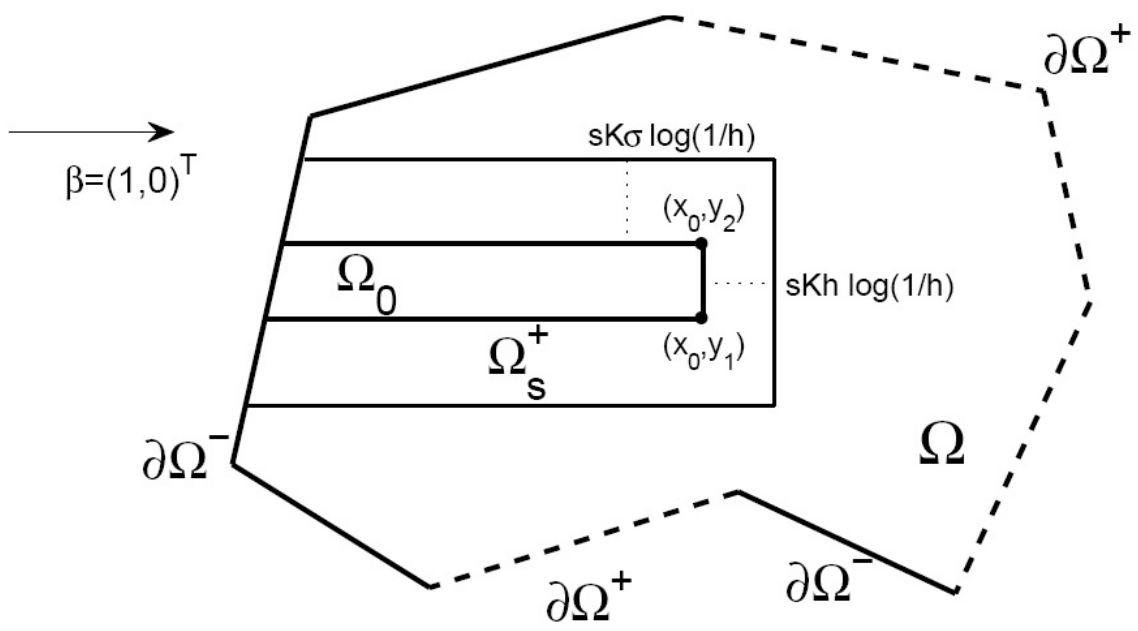

FiguRE 1. Sketch of $\Omega_{0}$ and $\Omega_{s}^{+}$

1.2. Main Result. The main goal of this paper is to obtain weighted a priori error estimates, where the weight $\omega$ is $O(1)$ on

$$
\Omega_{0}=\left(-\infty, x_{0}\right] \times\left[y_{1}, y_{2}\right] \cap \Omega
$$

and decays exponentially outside of a slightly larger subdomain (cf. Figure 1).

More precisely, the weight $\omega$ is a positive function with the following properties:

$$
\begin{aligned}
C_{1} \leq \omega(x, y) & \leq C_{2}, & & \text { for }(x, y) \in \Omega_{0}, \\
|\omega(x, y)| & \leq C e^{-\left(x-x_{0}\right) /(K h)}, & & \text { for } x \geq x_{0}+h, \\
|\omega(x, y)| & \leq C e^{-\left(y-y_{2}\right) /(K \sigma)}, & & \text { for } y \geq y_{2}+h, \\
|\omega(x, y)| & \leq C e^{-\left(y_{1}-y\right) /(K \sigma)}, & & \text { for } y \leq y_{1}-h .
\end{aligned}
$$

Here $C_{1}$ and $C_{2}$ are two fixed positive constants, $K>1$ is a sufficiently large number, and $\sigma$, the size of the crosswind layer, is

$$
\sigma:=\max \left(\varepsilon^{1 / 2}, h^{3 / 4}\right) .
$$

Theorem 1.1. For every $u \in H^{1}(\Omega)$ and $U \in V_{h}$ that satisfy (1.3) with $\varepsilon \leq h$, there exists a constant $C$ independent of $u$ and $U$, such that

$$
h^{1 / 2}\left\|\omega(u-U)_{x}\right\|_{L^{2}(\Omega)}+h^{3 / 4}\left\|\omega(u-U)_{y}\right\|_{L^{2}(\Omega)}+Q(u-U) \leq C \min _{\chi \in V_{h}} L(u-\chi),
$$

where

$$
\begin{aligned}
Q^{2}(v):= & \varepsilon\|\omega \nabla v\|_{L^{2}(\Omega)}^{2}+\frac{1}{2}\left\|\left(\omega\left|\omega_{x}\right|\right)^{1 / 2} v\right\|_{L^{2}(\Omega)}^{2}+\|\omega v\|_{L^{2}(\Omega)}^{2} \\
& +h^{2} \sum_{e \in \mathcal{E}_{h}^{0}}\left(\left\|\omega\left[v_{x}\right]\right\|_{L^{2}(e)}^{2}+\left\|\omega\left[v_{y}\right]\right\|_{L^{2}(e)}^{2}\right) \\
& +\frac{1}{2}\left\|\omega v\left|n_{x}\right|^{1 / 2}\right\|_{L^{2}(\partial \Omega)}^{2}+h^{1 / 2}\left\|\omega v n_{y}\right\|_{L^{2}(\partial \Omega)}^{2}+\gamma_{b c} \varepsilon h^{-1}\|\omega v\|_{L^{2}(\partial \Omega)}^{2}
\end{aligned}
$$


and

$$
L^{2}(v):=h\|\omega \nabla v\|_{L^{2}(\Omega)}^{2}+h^{-1}\|\omega v\|_{L^{2}(\Omega)}^{2}+h^{2} \sum_{e \in \mathcal{E}_{h}}\left(\left\|\omega\left[v_{x}\right]\right\|_{L^{2}(e)}^{2}+\left\|\omega\left[v_{y}\right]\right\|_{L^{2}(e)}^{2}\right) .
$$

We point out that by penalizing the crosswind derivative (i.e. by including the term $J_{\perp}$ in the method) we reduce the size of the crosswind layer for piecewise linear elements to order $\sigma=\max \left(h^{3 / 4}, \varepsilon^{1 / 2}\right)$ instead of order $\sigma=h^{1 / 2}$.

To give an application of the above result, let $\Omega_{0}$ be as in (1.4) and define

$$
\Omega_{s}^{+}=\left\{x \leq x_{0}+s K h|\log h|, y_{1}-s K \sigma|\log h| \leq y \leq y_{2}+s K \sigma|\log h|\right\} \cap \Omega .
$$

Corollary 1.2. Under the assumptions of Theorem 1.1 and assuming $u \in H^{t}(\Omega)$, there exists a constant $C$ independent of $u$ and $h$ such that for any $s>0$,

$$
\begin{gathered}
h^{1 / 2}\left\|(u-U)_{x}\right\|_{L^{2}\left(\Omega_{0}\right)}+h^{3 / 4}\left\|(u-U)_{y}\right\|_{L^{2}\left(\Omega_{0}\right)}+\|u-U\|_{L^{2}\left(\Omega_{0}\right)} \\
\leq C h^{r_{u}-\frac{1}{2}}\|u\|_{H^{r_{u}\left(\Omega_{s}^{+}\right)}}+C h^{s+r_{u}-\frac{1}{2}}\|u\|_{\left.H^{r_{u}(\Omega)}\right)}
\end{gathered}
$$

with $r_{u}=\min (2, t)$.

Remark 1 . Note that in the above estimate $r_{u}$ can be chosen to take different values in the different subdomains in the presence of singularities.

The rest of the paper is organized as follows. The next three sections are devoted to the proof of Theorem 1.1 for the piecewise linear case and constitute the main part of the paper. In Section 2 and Section 3, we collect some preliminary results which are necessary in order to carry out the proof of the Theorem 1.1. The proofs of Theorem 1.1 and Corollary 1.2 are presented in Section 4. The last section addresses the possible extensions, generalizations, and concluding remarks. Finally in the Appendix, we provide the proofs of the technical results stated in Section 3.

\section{Preliminary Results}

In this section we collect some results we require in our analysis. First we recall the standard trace and inverse inequalities. The proofs can be found in many textbooks on finite elements (cf. [6]).

2.1. Trace, inverse and interpolation inequalities. For $T \in \mathcal{T}_{h}$ and $v \in H^{1}(T)$ we have

$$
\|v\|_{L^{2}(\partial T)} \leq C_{t r}\left(h^{-1 / 2}\|v\|_{L^{2}(T)}+h^{1 / 2}\|\nabla v\|_{L^{2}(T)}\right),
$$

where $C_{t r}$ is independent of $T$ and $v$.

If $v \in \mathbb{P}^{1}(T)$, then

$$
\begin{gathered}
\|\nabla v\|_{L^{2}(T)} \leq C_{i n v} h^{-1}\|v\|_{L^{2}(T)}, \\
\|v\|_{L^{2}(\partial T)} \leq C_{i n v} h^{-1 / 2}\|v\|_{L^{2}(T)},
\end{gathered}
$$

where $C_{i n v}$ is independent of $h$ and $v$.

Let $T \in \mathcal{T}_{h}$ and $v \in H^{2}(T)$, then the interpolation inequality reads

$$
\|\nabla v\|_{L^{2}(T)} \leq C\left(h^{-1}\|v\|_{L^{2}(T)}+h\left\|D^{2} v\right\|_{L^{2}(T)}\right),
$$

for some constant $C$ independent of $T$ and $v$. 
2.2. The weight function. In addition to the properties $\omega$ described above, we assume that $\omega$ satisfies,

$$
\begin{aligned}
\omega_{x}(x, y) & <0, \text { for all }(x, y) \in \Omega \\
\left|D_{x}^{\alpha} D_{y}^{\beta} \omega\right| & \leq C K^{-\alpha} h^{-\alpha} K^{-\beta} \sigma^{-\beta} \omega, \text { for } \alpha+\beta \leq 2, \\
\left|D_{x}^{\alpha} D_{y}^{\beta} \omega\right| & \leq C K^{1-\alpha} h^{1-\alpha} K^{-\beta} \sigma^{-\beta}\left|\omega_{x}\right|, \text { for } \alpha \geq 1, \alpha+\beta \leq 2, \\
R O(S, \omega)+R O\left(S, \omega_{x}\right) & \leq C_{\omega}, \text { for any ball } S \text { of radius } K h
\end{aligned}
$$

where $R O(S, v)=\max _{\mathbf{x} \in S}|v(\mathbf{x})| / \min _{\mathbf{x} \in S}|v(\mathbf{x})|$. The explicit construction of such a function is given in [14]. The last property of $\omega$ enables us to apply the inverse inequalities (2.2) and (2.3) to functions of the form $\omega v$, with $v \in \mathbb{P}^{1}(T)$. For example,

$$
\begin{aligned}
\|\omega \nabla v\|_{L^{2}(T)} & \leq \max _{\mathbf{x} \in T} \omega(\mathbf{x})\|\nabla v\|_{L^{2}(T)} \leq \max _{\mathbf{x} \in T} \omega(\mathbf{x}) C_{i n v} h^{-1}\|v\|_{L^{2}(T)} \\
& \leq C_{i n v} C_{\omega} \min _{\mathbf{x} \in T} \omega(\mathbf{x}) h^{-1}\|v\|_{L^{2}(T)} \leq C_{i n v} C_{\omega} h^{-1}\|\omega v\|_{L^{2}(T)},
\end{aligned}
$$

and similarly

$$
\|\omega v\|_{L^{2}(\partial T)} \leq C_{i n v} C_{\omega} h^{-1 / 2}\|\omega v\|_{L^{2}(T)} .
$$

Finally, we would like to note that in the subsequent analysis it is important that $\omega^{-1}=\frac{1}{\omega}$ has the same properties (2.5)-(2.8) as $\omega$. For example,

$$
\left|D_{x} \omega^{-1}\right|=\left|\omega^{-2} D_{x} \omega\right| \leq \omega^{-2} C K^{-1} h^{-1} \omega=C K^{-1} h^{-1} \omega^{-1}
$$

and for any ball of radius $K h$,

$$
R O\left(S, \omega^{-1}\right)=\frac{\max _{\mathbf{x} \in T}[\omega(\mathbf{x})]^{-1}}{\min _{\mathbf{x} \in T}[\omega(\mathbf{x})]^{-1}}=\frac{\frac{1}{\min _{\mathbf{x} \in T} \omega(\mathbf{x})}}{\frac{1}{\max _{\mathbf{x} \in T} \omega(\mathbf{x})}}=R O(S, \omega) \leq C_{\omega} .
$$

2.3. Quasi-interpolant operator. Next we introduce a quasi-interpolant operator which is similar to the Clément operator [7]. The properties of this interpolant are essential to carry out the error analysis of the CIP method; see $[2,3,4]$. In this paper, we use the quasi-interpolant $\Pi$ presented in [4]. For simplicity we only consider the piecewise linear case. The value of $\Pi v$ at a node $\mathbf{x}_{i}$ of our finite element space $V_{h}$ (i.e. the nodes defined by the Lagrangian polynomial basis) is given by

$$
\Pi v\left(\mathbf{x}_{i}\right):=\left.\frac{1}{N_{i}} \sum_{\left\{T: \mathbf{x}_{i} \in T\right\}} v\right|_{T}\left(\mathbf{x}_{i}\right) .
$$

Here $N_{i}$ is the number of triangles in $\mathcal{T}_{h}$ that contain $\mathbf{x}_{i}$. One of the properties of $\Pi$ we will use in the subsequent analysis is the following $L^{2}$-stability result

$$
\|\Pi v\|_{L^{2}(\tau)} \leq C\|v\|_{L^{2}(\tau)}, \quad \forall v \in L^{2}(\tau) .
$$

\section{TEChNiCAL RESUlts}

In this section we state several results in the form of lemmas, which we require in order the carry out the proof of the main result. The proofs of these lemmas are quite technical and are given in the Appendix. 
3.1. Weighted estimates for a quasi-interpolant operator. The first result is a weighted version of Lemma 3.1 in [4]. This result is important in our presentation and will be used often. This type of estimate is also used in the a posteriori error estimation for non-conforming finite element methods and can be considered to be of independent interest.

Lemma 3.1. Let $U \in V_{h}$ and the operator $\Pi$ be given by (2.13). There exists a constant $C$ independent of $U, h$, and $K$ such that,

$$
\begin{gathered}
\left\|\omega\left(U_{x}-\Pi\left(U_{x}\right)\right)\right\|_{L^{2}(\Omega)}^{2} \leq C h \sum_{e \in \mathcal{E}_{h}^{0}}\left\|\omega\left[U_{x}\right]\right\|_{L^{2}(e)}^{2}, \\
\left\|\omega^{-1}\left(\omega^{2} U_{x}-\Pi\left(\omega^{2} U_{x}\right)\right)\right\|_{L^{2}(\Omega)}^{2} \leq C h \sum_{e \in \mathcal{E}_{h}^{0}}\left\|\omega\left[U_{x}\right]\right\|_{L^{2}(e)}^{2}+C K^{-2}\left\|\omega U_{x}\right\|_{L^{2}(\Omega)}^{2},
\end{gathered}
$$

and

$$
\begin{aligned}
& h^{2} \sum_{T \in \mathcal{T}_{h}}\left\|\omega^{-1} \nabla\left(\omega^{2} U_{x}-\Pi\left(\omega^{2} U_{x}\right)\right)\right\|_{L^{2}(T)}^{2} \\
& \quad \leq C h \sum_{e \in \mathcal{E}_{h}^{0}}\left\|\omega\left[U_{x}\right]\right\|_{L^{2}(e)}^{2}+C K^{-2}\left\|\omega U_{x}\right\|_{L^{2}(\Omega)}^{2} .
\end{aligned}
$$

3.2. Estimates for the crosswind derivative. The next result is special for the piecewise linear case. This lemma allows us to prove that in piecewise linear case the size of the crosswind layer is of order $\sigma=\max \left(h^{3 / 4}, \varepsilon^{1 / 2}\right)$. We would like to point out that the result below holds true for any function in $V_{h}$ and does not use the fact that $U$ is the approximate solution.

Lemma 3.2. Let $U \in V_{h}$. There exists a constant $C$ independent of $U$ and $h$, such that

$$
h^{3 / 2}\left\|\omega U_{y}\right\|_{L^{2}(\Omega)}^{2} \leq C\left(h^{2} \sum_{e \in \mathcal{E}_{h}^{0}}\left\|\omega\left[U_{y}\right]\right\|_{L^{2}(e)}^{2}+\|\omega U\|_{L^{2}(\Omega)}^{2}+h^{1 / 2}\left\|\omega U n_{y}\right\|_{L^{2}(\partial \Omega)}^{2}\right) .
$$

3.3. Estimates for the streamline derivative. In the next result we will give an estimate for the upwind term. In contrast to the above result, this lemma makes a strong use of the fact that $U$ is an approximate solution to (1.1). One of the main technical tools used to prove the following lemma is the weighted quasi-interpolant results in Lemma 3.1.

Lemma 3.3. Let $U \in V_{h}$ and $u \in H^{1}(\Omega)$ satisfy (1.3). There is a constant $C$ independent of $U, u$, and $h$, such that

$$
h\left\|\omega U_{x}\right\|_{L^{2}(\Omega)}^{2} \leq C\left(Q^{2}(U)+L^{2}(u)\right) .
$$

3.4. Superapproximation. The following superapproximation result is similar to the superapproximation result in [14] and [12]. The difference from the above mentioned superapproximation results is that instead of a local interpolant operator we here use a global $L^{2}$-projection. Because of this, the lemma has an independent interest. The orthogonal properties of the $L^{2}$-projection will be essential in our proof of the theorem. Indeed, this is what allows us to control the convective term by the gradient jump operator. 
Lemma 3.4. Let $U \in V_{h}$ and $u \in H^{1}(\Omega)$ satisfy (1.3). Let $P: L^{2}(\Omega) \rightarrow V_{h}$ denote the $L^{2}$-projection defined by

$$
\int_{\Omega} P v \chi d x=\int_{\Omega} v \chi d x, \quad \forall \chi \in V_{h} .
$$

Set $E=\omega^{2} U-P\left(\omega^{2} U\right)$. There exists a constant $C$ independent of $U, u, h$, and $K$, such that

$$
h^{-1}\left\|\omega^{-1} E\right\|_{L^{2}(\Omega)}^{2}+h\left\|\omega^{-1} \nabla E\right\|_{L^{2}(\Omega)}^{2} \leq C K^{-1}\left(Q^{2}(U)+L^{2}(u)\right) .
$$

Remark 2. By using the trace inequality (2.1) and property of $\omega$ (2.8), we may include $\left\|\omega^{-1} E\right\|_{L^{2}(\partial \Omega)}$ on the left hand side of Lemma 3.4, since,

$$
\begin{aligned}
\left\|\omega^{-1} E\right\|_{L^{2}(\partial \Omega)}^{2} & \leq \sum_{T \in \mathcal{T}_{h}} \min _{\mathbf{x} \in T}[\omega(\mathbf{x})]^{-2}\|E\|_{L^{2}(\partial T)}^{2} \\
& \leq C_{t r}^{2} \sum_{T \in \mathcal{T}_{h}} \min _{\mathbf{x} \in T}[\omega(\mathbf{x})]^{-2}\left(h^{-1}\|E\|_{L^{2}(T)}^{2}+h\|\nabla E\|_{L^{2}(T)}^{2}\right) \\
& \leq C_{t r}^{2} C_{\omega}^{2}\left(h^{-1}\left\|\omega^{-1} E\right\|_{L^{2}(\Omega)}^{2}+h\left\|\omega^{-1} \nabla E\right\|_{L^{2}(\Omega)}^{2}\right) .
\end{aligned}
$$

The last result states an important inequality which we will use in the second part of the proof of Theorem 1.1. The main technical tool used to prove this lemma is the above superappoximation result, Lemma 3.4.

Lemma 3.5. Let $U \in V_{h}$ and $u \in H^{1}(\Omega)$ satisfy (1.3). There exist constants $C$ and $\delta$, the latter may be chosen arbitrary small, independent of $U, u, h$, and $K$, and a constant $C_{\delta}$, which depends on $\delta$, but not on $U, u, h$, and $K$, such that

$$
B\left(U, \omega^{2} U\right) \leq C K^{-1} Q^{2}(U)+C \delta\left(Q^{2}(U)+h\left\|\omega U_{x}\right\|_{L^{2}(\Omega)}^{2}\right)+C_{\delta} L^{2}(u) .
$$

\section{Proof of Theorem 1.1 And Corollary 1.2}

After collecting all the necessary technical results in the previous section, we are ready to present a proof of Theorem 1.1. We prove the theorem in several steps.

\section{Proof. Step 1: Reduction to weighted stability.}

In this step we will prove that it is sufficient to show that

$$
Q(U) \leq C_{\delta} L(u)+C \delta h^{1 / 2}\left\|\omega U_{x}\right\|_{L^{2}(\Omega)},
$$

for all $U \in V_{h}$ and $u \in H^{1}(\Omega)$ that satisfy (1.3). Here $\delta$ is a constant that can be made as small as required.

By the triangle inequality, we have

$$
\begin{aligned}
& h^{1 / 2}\left\|\omega(U-u)_{x}\right\|_{L^{2}(\Omega)}+h^{3 / 4}\left\|\omega(U-u)_{y}\right\|_{L^{2}(\Omega)}+Q(U-u) \\
& \leq h^{1 / 2}\left\|\omega(U-\chi)_{x}\right\|_{L^{2}(\Omega)}+h^{3 / 4}\left\|\omega(U-\chi)_{y}\right\|_{L^{2}(\Omega)}+Q(U-\chi)+C L(u-\chi) .
\end{aligned}
$$

We have used that

$$
h^{1 / 2}\left\|\omega(u-\chi)_{x}\right\|_{L^{2}(\Omega)}+h^{3 / 4}\left\|\omega(u-\chi)_{y}\right\|_{L^{2}(\Omega)}+Q(u-\chi) \leq C L(u-\chi),
$$

which follows from the properties of $\omega$, the assumption $\varepsilon \leq h$, and the trace inequality (2.10). Hence, it is enough to show that for any $\chi \in V_{h}$,

$$
h^{1 / 2}\left\|\omega(U-\chi)_{x}\right\|_{L^{2}(\Omega)}+h^{3 / 4}\left\|\omega(U-\chi)_{y}\right\|_{L^{2}(\Omega)}+Q(U-\chi) \leq C L(u-\chi) .
$$


We note that if $\chi \in V_{h}$, then $\tilde{U}:=U-\chi \in V_{h}, \tilde{u}:=u-\chi \in H^{1}(\Omega)$ and

$$
B(\tilde{U}-\tilde{u}, v)=0, \quad \forall v \in V_{h} .
$$

Therefore, it is enough to show that

$$
h^{1 / 2}\left\|\omega \tilde{U}_{x}\right\|_{L^{2}(\Omega)}+h^{3 / 4}\left\|\omega \tilde{U}_{y}\right\|_{L^{2}(\Omega)}+Q(\tilde{U}) \leq C L(\tilde{u}) .
$$

In view of Lemma $3.2, h^{3 / 4}\left\|\omega \tilde{U}_{y}\right\|_{L^{2}(\Omega)} \leq C Q(\tilde{U})$. Moreover, using Lemma 3.3, it is sufficient to show

$$
Q(\tilde{U}) \leq C_{\delta} L(\tilde{u})+C \delta h^{1 / 2}\left\|\omega \tilde{U}_{x}\right\|_{L^{2}(\Omega)},
$$

where $\delta$ is a constant that can be made as small as desired. Using $U$ and $u$ instead of $\tilde{U}$ and $\tilde{u}$ we see that we need to show (4.1) for all $U \in V_{h}$ and $u \in H^{1}(\Omega)$ that satisfy (1.3).

Step 2: Relating $Q^{2}(U)$ to $B\left(U, \omega^{2} U\right)$.

In this step we will show that

$$
Q^{2}(U)=B\left(U, \omega^{2} U\right)-2 \varepsilon \int_{\Omega} U \omega \nabla \omega \cdot \nabla U-2 \varepsilon \int_{\partial \Omega}\left(\frac{\partial U}{\partial n} \omega^{2} U+U^{2} \omega \frac{\partial \omega}{\partial n}\right) d s .
$$

Recalling the definition (1.2), we have

$$
\begin{aligned}
B\left(U, \omega^{2} U\right) & =\varepsilon A\left(U, \omega^{2} U\right)+M\left(U, \omega^{2} U\right)+J_{\|}\left(U, \omega^{2} U\right)+J_{\perp}\left(U, \omega^{2} U\right) \\
& =\varepsilon\|\omega \nabla U\|_{L^{2}(\Omega)}^{2}+2 \varepsilon \int_{\Omega} U \omega \nabla \omega \cdot \nabla U \\
& -\varepsilon \int_{\partial \Omega}\left(\frac{\partial U}{\partial n} \omega^{2} U+\frac{\partial\left(\omega^{2} U\right)}{\partial n} U\right) d s+\frac{\varepsilon \gamma_{b c}}{h}\|\omega U\|_{L^{2}(\partial \Omega)}^{2} \\
& +\int_{\Omega} U_{x} \omega^{2} U+\|\omega U\|_{L^{2}(\Omega)}^{2}+\int_{\partial \Omega^{-}} \omega^{2} U^{2}\left|n_{x}\right| d s \\
& +h^{2} \sum_{e \in \mathcal{E}_{h}^{0}} \int_{e}\left(\left[U_{x}\right]\left[\left(\omega^{2} U\right)_{x}\right]+\left[U_{y}\right]\left[\left(\omega^{2} U\right)_{y}\right]\right) d s+h^{1 / 2}\left\|\omega U\left|n_{y}\right|\right\|_{L^{2}(\partial \Omega)}^{2} .
\end{aligned}
$$

Let us first treat the first term in $M\left(U, \omega^{2} U\right)$, namely $\int_{\Omega} U_{x} \omega^{2} U$. By integration by parts and using that $\omega_{x}<0$, we have

$$
\begin{aligned}
\int_{\Omega} U_{x} \omega^{2} U & =-\int_{\Omega} U^{2} \omega \omega_{x}+\frac{1}{2} \int_{\partial \Omega} \omega^{2} U^{2} n_{x} d s \\
& =\left\|\left(\omega\left|\omega_{x}\right|\right)^{1 / 2} U\right\|_{L^{2}(\Omega)}^{2}+\frac{1}{2} \int_{\partial \Omega^{-}} \omega^{2} U^{2} n_{x} d s+\frac{1}{2} \int_{\partial \Omega \backslash \partial \Omega^{-}} \omega^{2} U^{2} n_{x} d s .
\end{aligned}
$$

Because $n_{x}<0$ on $\partial \Omega^{-}$, we have

$$
\begin{aligned}
\int_{\partial \Omega^{-}} \omega^{2} U^{2}\left|n_{x}\right| d s & +\frac{1}{2} \int_{\partial \Omega^{-}} \omega^{2} U^{2} n_{x} d s+\frac{1}{2} \int_{\partial \Omega \backslash \partial \Omega^{-}} \omega^{2} U^{2} n_{x} d s \\
& =-\frac{1}{2} \int_{\partial \Omega^{-}} \omega^{2} U^{2} n_{x} d s+\frac{1}{2} \int_{\partial \Omega \backslash \partial \Omega^{-}} \omega^{2} U^{2} n_{x} d s \\
& =\frac{1}{2}\left\|\omega U\left|n_{x}\right|^{1 / 2}\right\|_{L^{2}(\partial \Omega)}^{2} .
\end{aligned}
$$


Since, $\omega$ is smooth and $U$ is continuous, the jump terms $\left[\left(\omega^{2}\right)_{x} U\right]$ and $\left[\left(\omega^{2}\right)_{y} U\right]$ vanish and we have

$$
\begin{aligned}
& \int_{e}\left(\left[U_{x}\right]\left[\left(\omega^{2} U\right)_{x}\right]+\left[U_{y}\right]\left[\left(\omega^{2} U\right)_{y}\right]\right) d s \\
& =\int_{e}\left(\left[U_{x}\right]\left[\left(\omega^{2}\right)_{x} U+\omega^{2} U_{x}\right]+\left[U_{y}\right]\left[\left(\omega^{2}\right)_{y} U+\omega^{2} U_{y}\right]\right) d s \\
& =\left\|\omega\left[U_{x}\right]\right\|_{L^{2}(e)}^{2}+\left\|\omega\left[U_{y}\right]\right\|_{L^{2}(e)}^{2} .
\end{aligned}
$$

Taking into account the above arguments, we have shown (4.3).

Step 3: Initial Estimate for $Q^{2}(U)$.

In this step we will bound the last two terms on the right hand side of (4.3) to show

$$
Q^{2}(U) \leq C B\left(U, \omega^{2} U\right)
$$

For the second term appearing in the right hand side of (4.3) we note that

$$
\int_{\Omega} U \omega \nabla \omega \cdot \nabla U=\int_{\Omega} U \omega\left(\omega_{x} U_{x}+\omega_{y} U_{y}\right),
$$

hence by the Cauchy-Schwarz inequality, (2.6), and using that $\varepsilon \leq h$,

$$
\begin{aligned}
\varepsilon \int_{\Omega} U \omega \omega_{x} U_{x} & \leq \varepsilon\left\|\omega U_{x}\right\|_{L^{2}(\Omega)}\left\|\omega_{x} U\right\|_{L^{2}(\Omega)} \\
& \leq \varepsilon\left\|\omega U_{x}\right\|_{L^{2}(\Omega)} C h^{-1 / 2} K^{-1 / 2}\left\|\left(\omega\left|\omega_{x}\right|\right)^{1 / 2} U\right\|_{L^{2}(\Omega)} \\
& \leq C K^{-1 / 2}\left(\varepsilon\|\omega \nabla U\|_{L^{2}(\Omega)}^{2}+\varepsilon h^{-1}\left\|\left(\omega\left|\omega_{x}\right|\right)^{1 / 2} U\right\|_{L^{2}(\Omega)}^{2}\right) \\
& \leq C K^{-1 / 2} Q^{2}(U) .
\end{aligned}
$$

Similarly, by (2.6) and using that by definition (1.5) $\sigma=\max \left(\varepsilon^{1 / 2}, h^{3 / 4}\right)$ and therefore $\varepsilon \leq \sigma^{2}$,

$$
\begin{aligned}
\varepsilon \int_{\Omega} \omega U \omega_{y} U_{y} & \leq \varepsilon\left\|\omega U_{y}\right\|_{L^{2}(\Omega)}\left\|\omega_{y} U\right\|_{L^{2}(\Omega)} \leq \varepsilon\left\|\omega U_{y}\right\|_{L^{2}(\Omega)} C \sigma^{-1} K^{-1}\|\omega U\|_{L^{2}(\Omega)} \\
& \leq C K^{-1}\left(\varepsilon\|\omega \nabla U\|_{L^{2}(\Omega)}^{2}+\varepsilon \sigma^{-2}\|\omega U\|_{L^{2}(\Omega)}^{2}\right) \leq C K^{-1} Q^{2}(U) .
\end{aligned}
$$

Combining, (4.5) and (4.6), we obtain

$$
\varepsilon \int_{\Omega} \omega U \nabla \omega \cdot \nabla U \leq C K^{-1 / 2} Q^{2}(U) .
$$

Next we will estimate the boundary term. By the Cauchy-Schwarz inequality, the property (2.8) of $\omega$, and the trace inequality (2.10),

$$
\begin{aligned}
\varepsilon \int_{\partial \Omega} \frac{\partial U}{\partial n} \omega^{2} U d s & \leq \varepsilon\left\|\omega \frac{\partial U}{\partial n}\right\|_{L^{2}(\partial \Omega)}\|\omega U\|_{L^{2}(\partial \Omega)} \\
& \leq \varepsilon C_{\omega} C_{i n v} h^{-1 / 2}\|\omega \nabla U\|_{L^{2}(\Omega)}\|\omega U\|_{L^{2}(\partial \Omega)} \\
& \leq \frac{\varepsilon}{4}\|\omega \nabla U\|_{L^{2}(\Omega)}^{2}+\varepsilon \frac{C_{\omega}^{2} C_{i n v}^{2}}{h}\|\omega U\|_{L^{2}(\partial \Omega)}^{2} .
\end{aligned}
$$

Also, using (2.6) and (2.8), we see that

$$
\varepsilon \int_{\partial \Omega} U^{2} \omega \frac{\partial \omega}{\partial n} d s \leq C K^{-1} \frac{\varepsilon}{h}\|\omega U\|_{L^{2}(\partial \Omega)}^{2} .
$$


Therefore, if $\gamma_{b c}>2 C_{i n v}^{2} C_{\omega}^{2}$ and by taking $K$ large enough, we have

$$
2 \varepsilon \int_{\partial \Omega}\left(\frac{\partial U}{\partial n} \omega^{2} U+U^{2} \omega \frac{\partial \omega}{\partial n}\right) d s \leq \frac{1}{2} Q^{2}(U) .
$$

If we substitute (4.8) and (4.7) into (4.3) and choose $K$ sufficienlty large we have (4.4).

Step 4: Final estimate for $Q^{2}(U)$.

Applying Lemma 3.5 to the right hand side of (4.4), we obtain

$$
Q^{2}(U) \leq C K^{-1} Q^{2}(U)+C \delta\left(Q^{2}(U)+h\left\|\omega U_{x}\right\|_{L^{2}(\Omega)}^{2}\right)+C_{\delta} L^{2}(u) .
$$

Choosing $K$ large and $\delta$ small, shows (4.1). This proves the theorem.

Now we will prove Corollary 1.2

Proof. By Theorem 1.1 and the triangle inequality, for any $\chi \in V_{h}$ we have,

$$
\begin{aligned}
& h^{1 / 2}\left\|(u-U)_{x}\right\|_{L^{2}\left(\Omega_{0}\right)}+h^{3 / 4}\left\|(u-U)_{y}\right\|_{L^{2}\left(\Omega_{0}\right)}+\|u-U\|_{L^{2}\left(\Omega_{0}\right)} \\
& \leq C\left(h^{1 / 2}\left\|\omega(u-U)_{x}\right\|_{L^{2}(\Omega)}+h^{3 / 4}\left\|\omega(u-U)_{y}\right\|_{L^{2}(\Omega)}+\|\omega(u-U)\|_{L^{2}(\Omega)}\right) \\
& \leq C L(u-\chi) \\
& \leq C\left(h^{1 / 2}\|\nabla(u-\chi)\|_{L^{2}\left(\Omega_{s}^{+}\right)}+h^{-1 / 2}\|u-\chi\|_{L^{2}\left(\Omega_{s}^{+}\right)}+h\left(\sum_{e \in \mathcal{E}_{h} \cap \Omega_{s}^{+}}\|\nabla(u-\chi)\|_{L^{2}(e)}^{2}\right)^{\frac{1}{2}}\right. \\
& +h^{1 / 2}\|\omega \nabla(u-\chi)\|_{L^{2}\left(\Omega \backslash \Omega_{s}^{+}\right)}+h^{-1 / 2}\|\omega(u-\chi)\|_{L^{2}\left(\Omega \backslash \Omega_{s}^{+}\right)} \\
& \left.+h\left(\sum_{e \in \mathcal{E}_{h} \cap \Omega \backslash \Omega_{s}^{+}}\|\omega \nabla(u-\chi)\|_{L^{2}(e)}^{2}\right)^{1 / 2}\right) .
\end{aligned}
$$

Taking $\chi=I_{h} u$ to be the Lagrange interpolant of $u$ and using the approximation theory together with the trace inequality $(2.1)$, we obtain

$$
\begin{gathered}
h^{1 / 2}\left\|\nabla\left(u-I_{h} u\right)\right\|_{L^{2}\left(\Omega_{s}^{+}\right)}+h^{-1 / 2}\left\|u-I_{h} u\right\|_{L^{2}\left(\Omega_{s}^{+}\right)}+h\left(\sum_{e \in \mathcal{E}_{h} \cap \Omega_{s}^{+}}\left\|\nabla\left(u-I_{h} u\right)\right\|_{L^{2}(e)}^{2}\right)^{\frac{1}{2}} \\
\leq C h^{r_{u}-\frac{1}{2}}\|u\|_{H^{r u}\left(\Omega_{s}^{+}\right)} .
\end{gathered}
$$

For the other terms using that $\omega=O\left(h^{s}\right)$ outside of $\Omega_{s}^{+}$, we have

$$
\begin{aligned}
& h^{1 / 2}\left\|\omega \nabla\left(u-I_{h} u\right)\right\|_{L^{2}\left(\Omega \backslash \Omega_{s}^{+}\right)}+h^{-1 / 2}\left\|\omega\left(u-I_{h} u\right)\right\|_{L^{2}\left(\Omega \backslash \Omega_{s}^{+}\right)} \\
& +h\left(\sum_{e \in \mathcal{E}_{h} \cap \Omega \backslash \Omega_{s}^{+}}\left\|\omega \nabla\left(u-I_{h} u\right)\right\|_{L^{2}(e)}^{2}\right)^{1 / 2} \leq \\
& C h^{s}\left(h^{1 / 2}\left\|\nabla\left(u-I_{h} u\right)\right\|_{L^{2}(\Omega)}+h^{-1 / 2}\left\|u-I_{h} u\right\|_{L^{2}(\Omega)}+h\left(\sum_{e \in \mathcal{E}_{h}}\left\|\nabla\left(u-I_{h} u\right)\right\|_{L^{2}(e)}^{2}\right)^{1 / 2}\right) .
\end{aligned}
$$


Similarly, by the trace inequality (2.1) and the approximation theory, we have

$$
\begin{aligned}
& h^{1 / 2}\left\|\nabla\left(u-I_{h} u\right)\right\|_{L^{2}(\Omega)}+h^{-1 / 2}\left\|u-I_{h} u\right\|_{L^{2}(\Omega)}+h\left(\sum_{e \in \mathcal{E}_{h}}\left\|\nabla\left(u-I_{h} u\right)\right\|_{L^{2}(e)}^{2}\right)^{\frac{1}{2}} \\
& \leq C\left(h^{1 / 2}\left\|\nabla\left(u-I_{h} u\right)\right\|_{L^{2}(\Omega)}+h^{-1 / 2}\left\|u-I_{h} u\right\|_{L^{2}(\Omega)}+h^{r_{u}-\frac{1}{2}}\left\|D^{r_{u}} u\right\|_{L^{2}(\Omega)}\right) \\
& \leq C h^{r_{u}-\frac{1}{2}}\|u\|_{H^{r_{u}}(\Omega)} .
\end{aligned}
$$

This proves the corollary.

\section{EXTENSIONS AND CONCLUDING REMARKS}

There are several extensions of the above analysis that are straightforward although technical. Below we will comment on some of the more important ones.

5.1. High-order elements. The extension of the present analysis to high-order elements is straightforward. In our analysis, only Lemma 3.2 makes strong use of the vanishing second derivative in an essential way. It seems that the crosswind jump term (i.e. $J_{\perp}$ ) is useful to control crosswind smearing only in the piecewise linear case. It remains unclear whether the crosswind stabilization for high-order elements has any effect. However, we can still prove a slightly weaker result than Theorem 1.1 of the form

$$
h^{1 / 2}\left\|\omega(u-U)_{x}\right\|_{L^{2}(\Omega)}+Q(u-U) \leq C \min _{\chi \in V_{h}} L(u-\chi),
$$

where we need to take $\sigma=h^{1 / 2}$. This means that we can only prove that the crosswind layer is of size $h^{1 / 2}$ rather than $\max \left(h^{3 / 4}, \varepsilon^{1 / 2}\right)$. It is not clear if this result is sharp for high-order elements.

Since the Lemma 3.2 does not hold for $k \geq 2$, in order to establish (5.1) one will need a slightly different superapproximation result of the form

$$
\begin{aligned}
h^{-1}\left\|\omega^{-1} E\right\|_{L^{2}(\Omega)}^{2} & +h\left\|\omega^{-1} \nabla E\right\|_{L^{2}(\Omega)}^{2} \\
& \leq C K^{-1}\left(\|\omega U\|_{L^{2}(\Omega)}^{2}+\left\|\left(\left|\omega \omega_{x}\right|\right)^{1 / 2} U\right\|_{L^{2}(\Omega)}^{2}\right) .
\end{aligned}
$$

5.2. $L^{\infty}$-norm estimates. In this paper we have presented weighted error estimates in $L^{2}$-norm. Using similar weighted stability estimates one can prove suboptimal $L^{\infty}$-norm estimates in regions away from boundary layers. More precisely, if we use the technique in [15], we can prove

$$
\|u-U\|_{L^{\infty}\left(\Omega_{0}\right)} \leq C h^{11 / 8},
$$

where $\Omega_{0} \subset \Omega$ and the distance from $\Omega_{0}$ to the outflow part of the boundary of $\Omega$ is at least $K h \log (1 / h))$ for a sufficiently large constant $K$.

5.3. No reaction term and variable convection. In our presentation, for simplicity, we assumed the reaction term in (1.1) is just $u$. However, if instead we have used the weight function presented in $[12,16]$, we could have shown the results for a more general problem

$$
\begin{aligned}
-\varepsilon \Delta u+\beta \cdot \nabla u+c u & =f & & \text { in } \Omega, \\
u & =0 & & \text { on } \partial \Omega,
\end{aligned}
$$

where $\beta \in\left[L^{\infty}(\Omega)\right]^{2}$ and $c \in L^{\infty}(\Omega), c(x) \geq 0$. 
5.4. Summary and concluding remarks. We have proved a weighted a priori error estimate for the CIP method applied to a convection dominated second order convection-diffusion equation.

We also proved that by including extra terms we can reduce the size of numerical layer in the crosswind direction. Typically the numerical layer in the upwind direction is of order $O(h \log 1 / h)$ whereas it is of order $O\left(h^{1 / 2} \log 1 / h\right)$ in the crosswind direction. In our analysis of the piecewise linear case by adding an extra penalty term and using $\sigma=\max \left(h^{3 / 4}, \varepsilon^{1 / 2}\right)$, we are able to reduce the size of the crosswind numerical layer to order $O\left(h^{3 / 4} \log 1 / h\right)$.

The reason we were able to accomplish this is that once Lemma 3.2 was established, we could use the superapproximation result, Lemma 3.4. The reason that Lemma 3.2 holds is that we have included the term $J_{\perp}$ in the definition of the CIP method. In other words, penalizing the jumps of the crosswind derivative allows us to reduce the size of the crosswind numerical layer to $\sigma=\max \left(\varepsilon^{1 / 2}, h^{3 / 4}\right)$ in the case of piecewise linear approximations. This argument is similar to the results of [14], where more crosswind diffusion was added in order to reduce the crosswind smear. Note however that in spite of the fact that the CIP-crosswind diffusion is weakly consistent this property does not seem to generalize to the case of higher order polynomial approximation.

These types of results are expected to be of interest in problems in fluid mechanics. For example, if similar results held for the CIP-method of [4] applied to the Oseen's equation it would mean that, away from boundary layers and singularities, solutions to the linearized equations of incompressible flow are accurately approximated by the CIP-method also in the high Reynolds number regime.

\section{Appendix: Technical proofs}

\subsection{Proof of Lemma 3.1.}

Proof. Let $\Delta(T)$ denote the closure of the set of triangles $T^{\prime} \in \mathcal{T}_{h}$ such that $T \cap T^{\prime} \neq$ $\emptyset$. We start by proving (3.1). By [4], for any piecewise polynomial function $p$

$$
\|p-\Pi p\|_{L^{2}(T)}^{2} \leq C h \sum_{e \in \mathcal{E}_{h}^{0}, e \subset \Delta(T)}\|[p]\|_{L^{2}(e)}^{2} .
$$

Using (2.8) and (6.1), we can estimate the left hand side of (3.1) as

$$
\begin{aligned}
\left\|\omega\left(U_{x}-\Pi\left(U_{x}\right)\right)\right\|_{L^{2}(\Omega)}^{2} & \leq \sum_{T \in \mathcal{T}_{h}} \max _{\mathbf{x} \in T}[\omega(\mathbf{x})]^{2}\left\|U_{x}-\Pi\left(U_{x}\right)\right\|_{L^{2}(T)}^{2} \\
& \leq C \sum_{T \in \mathcal{T}_{h}} \max _{\mathbf{x} \in T}[\omega(\mathbf{x})]^{2} h \sum_{e \in \mathcal{E}_{h}^{0}, e \subset \Delta(T)}\left\|\left[U_{x}\right]\right\|_{L^{2}(e)}^{2} \\
& \leq C C_{\omega}^{2} h \sum_{e \in \mathcal{E}_{h}^{0}}\left\|\omega\left[U_{x}\right]\right\|_{L^{2}(e)}^{2},
\end{aligned}
$$

which proves (3.1).

Next we will establish (3.2). By the triangle inequality,

$$
\begin{aligned}
\left\|\omega^{-1}\left(\omega^{2} U_{x}-\Pi\left(\omega^{2} U_{x}\right)\right)\right\|_{L^{2}(\Omega)}^{2} & \leq C\left(\left\|\omega^{-1}\left(\Pi\left(\overline{\omega^{2}} U_{x}\right)-\Pi\left(\omega^{2} U_{x}\right)\right)\right\|_{L^{2}(\Omega)}^{2}\right. \\
& +\left\|\omega^{-1}\left(\Pi\left(\overline{\omega^{2}} U_{x}\right)-\overline{\omega^{2}} U_{x}\right)\right\|_{L^{2}(\Omega)}^{2} \\
& \left.+\left\|\omega^{-1}\left(\overline{\omega^{2}} U_{x}-\omega^{2} U_{x}\right)\right\|_{L^{2}(\Omega)}^{2}\right)=C\left(I_{1}+I_{2}+I_{3}\right),
\end{aligned}
$$


where $\overline{\omega^{2}}=\frac{1}{|T|} \int_{T} \omega^{2}$ is the average of $\omega^{2}$ over each triangle.

Using (2.12) and the stability of $\Pi$ in the $L^{2}$-norm, we have

$$
\begin{aligned}
I_{1} & =\sum_{T \in \mathcal{T}_{h}}\left\|\omega^{-1}\left(\Pi\left(\overline{\omega^{2}} U_{x}\right)-\Pi\left(\omega^{2} U_{x}\right)\right)\right\|_{L^{2}(T)}^{2} \\
& \leq C \sum_{T \in \mathcal{T}_{h}} \min _{\mathbf{x} \in T}[\omega(\mathbf{x})]^{-2}\left\|\overline{\omega^{2}} U_{x}-\omega^{2} U_{x}\right\|_{L^{2}(T)}^{2} \\
& \leq C C_{\omega}^{2}\left\|\omega^{-1}\left(\overline{\omega^{2}} U_{x}-\omega^{2} U_{x}\right)\right\|_{L^{2}(\Omega)}^{2}=C C_{\omega}^{2} I_{3} .
\end{aligned}
$$

Thus, we need only to estimate $I_{2}$ and $I_{3}$. To estimate $I_{3}$ we use the fact that $\left\|\omega^{2}-\overline{\omega^{2}}\right\|_{L^{\infty}(T)} \leq C h\left\|\nabla\left(\omega^{2}\right)\right\|_{L^{\infty}(T)}$ and the properties of $\omega$ (2.6) and (2.12). Thus,

$$
\begin{aligned}
I_{3}= & \sum_{T \in \mathcal{T}_{h}}\left\|\omega^{-1}\left(\overline{\omega^{2}} U_{x}-\omega^{2} U_{x}\right)\right\|_{L^{2}(T)}^{2} \\
& \leq C \sum_{T \in \mathcal{T}_{h}} \min _{\mathbf{x} \in T}[\omega(\mathbf{x})]^{-2} h^{2}\left\|\nabla\left(\omega^{2}\right)\right\|_{L^{\infty}(T)}^{2}\left\|U_{x}\right\|_{L^{2}(T)}^{2} \\
& \leq C \sum_{T \in \mathcal{T}_{h}} \min _{\mathbf{x} \in T}[\omega(\mathbf{x})]^{-2} K^{-2}\left\|\omega^{4}\right\|_{L^{\infty}(T)}\left\|U_{x}\right\|_{L^{2}(T)}^{2} \\
& \leq C C_{\omega}^{4} \sum_{T \in \mathcal{T}_{h}} K^{-2}\left\|\omega U_{x}\right\|_{L^{2}(T)}^{2}=C C_{\omega}^{4} K^{-2}\left\|\omega U_{x}\right\|_{L^{2}(\Omega)}^{2}
\end{aligned}
$$

Thus, we are only left to estimate $I_{2}$. Again using (2.12), (6.1), and the triangle inequality, we have (6.4)

$$
\begin{aligned}
I_{2} & =\sum_{T \in \mathcal{T}_{h}}\left\|\omega^{-1}\left(\Pi\left(\overline{\omega^{2}} U_{x}\right)-\overline{\omega^{2}} U_{x}\right)\right\|_{L^{2}(T)}^{2} \\
& \left.\leq \sum_{T \in \mathcal{T}_{h}} \min _{\mathbf{x} \in T}[\omega(\mathbf{x})]^{-2} \| \Pi\left(\overline{\omega^{2}} U_{x}\right)-\overline{\omega^{2}} U_{x}\right) \|_{L^{2}(T)}^{2} \\
& \leq C \sum_{T \in \mathcal{T}_{h}} \min _{\mathbf{x} \in T}[\omega(\mathbf{x})]^{-2} h \sum_{e \in \mathcal{E}_{h}^{0}, e \subset \Delta(T)}\left\|\left[\overline{\omega^{2}} U_{x}\right]\right\|_{L^{2}(e)}^{2} \\
& \leq C h \sum_{T \in \mathcal{T}_{h}} \min _{\mathbf{x} \in T}[\omega(\mathbf{x})]^{-2} \sum_{e \in \mathcal{E}_{h}^{0}, e \subset \Delta(T)}\left(\left\|\left[\omega^{2} U_{x}\right]\right\|_{L^{2}(e)}^{2}+\left\|\left[\left(\overline{\omega^{2}}-\omega^{2}\right) U_{x}\right]\right\|_{L^{2}(e)}^{2}\right) .
\end{aligned}
$$

Since $\omega$ is smooth, $\left[\omega^{2} U_{x}\right]=\omega^{2}\left[U_{x}\right]$, and using $(2.12)$, we have

$$
\begin{aligned}
h \sum_{T \in \mathcal{T}_{h}} \min _{\mathbf{x} \in T}[\omega(\mathbf{x})]^{-2} \sum_{e \in \mathcal{E}_{h}^{0}, e \subset \Delta(T)}\left\|\omega^{2}\left[U_{x}\right]\right\|_{L^{2}(e)}^{2} & \leq C_{\omega}^{2} h \sum_{T \in \mathcal{T}_{h}} \sum_{e \in \mathcal{E}_{h}^{0}, e \subset \Delta(T)}\left\|\omega\left[U_{x}\right]\right\|_{L^{2}(e)}^{2} \\
& \leq h C C_{\omega}^{2} \sum_{e \in \mathcal{E}_{h}^{0}}\left\|\omega\left[U_{x}\right]\right\|_{L^{2}(e)}^{2} .
\end{aligned}
$$


To estimate the remaining term in $I_{2}$, we proceed similarly to (6.3) and use the inverse inequality (2.3) applied to $\left[U_{x}\right]$,

$$
\begin{aligned}
& h \sum_{T \in \mathcal{T}_{h}} \min _{\mathbf{x} \in T}[\omega(\mathbf{x})]^{-2} \sum_{e \in \mathcal{E}_{h}^{0}, e \subset \Delta(T)}\left\|\left[\left(\overline{\omega^{2}}-\omega^{2}\right) U_{x}\right]\right\|_{L^{2}(e)}^{2} \\
& \leq C h \sum_{T \in \mathcal{T}_{h}} \min _{\mathbf{x} \in T}[\omega(\mathbf{x})]^{-2} h^{2}\left\|\nabla\left(\omega^{2}\right)\right\|_{L^{\infty}(\Delta(T))}^{2} \sum_{e \in \mathcal{E}_{h}^{0}, e \subset \Delta(T)}\left\|\left[U_{x}\right]\right\|_{L^{2}(e)}^{2} \\
& \leq C C_{i n v}^{2} h \sum_{T \in \mathcal{T}_{h}} \min _{\mathbf{x} \in T}[\omega(\mathbf{x})]^{-2} K^{-2}\left\|\omega^{4}\right\|_{L^{\infty}(\Delta(T))} \sum_{T \subset \Delta(T)} h^{-1}\left\|U_{x}\right\|_{L^{2}(T)}^{2} \\
& \leq C C_{i n v}^{2} C_{\omega}^{4} K^{-2} \sum_{T \in \mathcal{T}_{h}} \sum_{T \subset \Delta(T)}\left\|\omega U_{x}\right\|_{L^{2}(T)}^{2} \\
& \leq C C_{i n v}^{2} C_{\omega}^{4} K^{-2} \sum_{T \in \mathcal{T}_{h}}\left\|\omega U_{x}\right\|_{L^{2}(T)}^{2}=C C_{i n v}^{2} C_{\omega}^{4} K^{-2}\left\|\omega U_{x}\right\|_{L^{2}(\Omega)}^{2} .
\end{aligned}
$$

Combining the above two estimates with estimates (6.2), (6.4), and (6.3), we prove (3.2).

Finally, to obtain (3.3), we apply (2.12), triangle inequality, and the inverse inequality to $\overline{\omega^{2}} U_{x}-\Pi\left(\omega^{2} U_{x}\right)$, we obtain,

$$
\begin{aligned}
& h^{2} \sum_{T \in \mathcal{T}_{h}}\left\|\omega^{-1} \nabla\left(\omega^{2} U_{x}-\Pi\left(\omega^{2} U_{x}\right)\right)\right\|_{L^{2}(T)}^{2} \\
& \leq C h^{2} \sum_{T \in \mathcal{T}_{h}} \min _{\mathbf{x} \in T}[\omega(\mathbf{x})]^{-2}\left\|\nabla\left(\omega^{2} U_{x}-\Pi\left(\omega^{2} U_{x}\right)\right)\right\|_{L^{2}(T)}^{2} \\
& \leq C h^{2} \sum_{T \in \mathcal{T}_{h}} \min _{\mathbf{x} \in T}[\omega(\mathbf{x})]^{-2}\left(\left\|\nabla\left(\omega^{2} U_{x}-\overline{\omega^{2}} U_{x}\right)\right\|_{L^{2}(T)}^{2}+\left\|\nabla\left(\overline{\omega^{2}} U_{x}-\Pi\left(\omega^{2} U_{x}\right)\right)\right\|_{L^{2}(T)}^{2}\right) \\
& \leq C \sum_{T \in \mathcal{T}_{h}} \min _{\mathbf{x} \in T}[\omega(\mathbf{x})]^{-2}\left(h^{2}\left\|\nabla\left(\omega^{2} U_{x}-\overline{\omega^{2}} U_{x}\right)\right\|_{L^{2}(T)}^{2}+C_{i n v}^{2}\left\|\overline{\omega^{2}} U_{x}-\Pi\left(\omega^{2} U_{x}\right)\right\|_{L^{2}(T)}^{2}\right) \\
& \leq C C_{\omega}^{2} \sum_{T \in \mathcal{T}_{h}} h^{2}\left\|\omega^{-1} \nabla\left(\left(\overline{\omega^{2}}-\omega^{2}\right) U_{x}\right)\right\|_{L^{2}(T)}^{2}+C C_{i n v}^{2} C_{\omega}^{2} \sum_{T \in \mathcal{T}_{h}} \| \omega^{-1}\left(\overline{\omega^{2}} U_{x}-\Pi\left(\omega^{2} U_{x}\right) \|_{L^{2}(T)}^{2}\right. \\
& \leq C C_{\omega}^{2} \sum_{T \in \mathcal{T}_{h}} h^{2}\left\|\omega^{-1} \nabla\left(\left(\overline{\omega^{2}}-\omega^{2}\right) U_{x}\right)\right\|_{L^{2}(T)}^{2}+C C_{i n v}^{2} C_{\omega}^{2}\left(I_{1}+I_{2}\right) .
\end{aligned}
$$

Since we already showed the desired estimate for $I_{1}$ and $I_{2}$, to finish the proof we use (2.6), (2.12), and noticing that $\nabla U_{x}=0$, we obtain,

$h^{2} \sum_{T \in \mathcal{T}_{h}}\left\|\omega^{-1} \nabla\left(\left(\overline{\omega^{2}}-\omega^{2}\right) U_{x}\right)\right\|_{L^{2}(T)}^{2}=h^{2} \sum_{T \in \mathcal{T}_{h}}\left\|2 \nabla \omega U_{x}\right\|_{L^{2}(T)}^{2} \leq C K^{-2} \sum_{T \in \mathcal{T}_{h}}\left\|\omega U_{x}\right\|_{L^{2}(T)}^{2}$.

This concludes the proof of the lemma.

\subsection{Proof of Lemma 3.2.}

Proof. Using the integration by parts and the fact that $U_{y}$ is piecewise constant,

$$
\left\|\omega U_{y}\right\|_{L^{2}(\Omega)}^{2}=\sum_{T \in \mathcal{T}_{h}} \int_{T} \omega^{2} U_{y}^{2}=\sum_{T \in \mathcal{T}_{h}}\left(-\int_{T} 2 \omega \omega_{y} U U_{y}+\int_{\partial T} \omega^{2} U U_{y} n_{y} d s\right)
$$


Using the property of $\omega$, namely (2.6), the Cauchy-Schwarz and arithmetic-geometric mean inequalities, we can estimate the first term on the right hand side by

$$
\begin{aligned}
-h^{3 / 2} \sum_{T \in \mathcal{T}_{h}} \int_{T} 2 \omega \omega_{y} U U_{y} & \leq C h^{3 / 4} K^{-1}\left\|\omega U_{y}\right\|_{L^{2}(\Omega)}\|\omega U\|_{L^{2}(\Omega)} \\
& \leq \frac{h^{3 / 2}}{4}\left\|\omega U_{y}\right\|_{L^{2}(\Omega)}^{2}+C K^{-2}\|\omega U\|_{L^{2}(\Omega)}^{2} .
\end{aligned}
$$

By the Cauchy-Schwarz and arithmetic-geometric mean inequalities, we can estimate the second term on the right hand side by

$$
\begin{aligned}
h^{3 / 2} \sum_{T \in \mathcal{T}_{h}} \int_{\partial T} \omega^{2} U U_{y} n_{y} d s & \leq h^{3 / 2} \sum_{e \in \mathcal{E}_{h}^{0}}\left\|\omega\left[U_{y}\right]\right\|_{L^{2}(e)}\|\omega U\|_{L^{2}(e)} \\
& +h^{3 / 2}\left\|\omega U n_{y}\right\|_{L^{2}(\partial \Omega)}\left\|\omega U_{y}\right\|_{L^{2}(\partial \Omega)} \\
& \leq \frac{1}{2} \sum_{e \in \mathcal{E}_{h}^{0}}\left(h^{2}\left\|\omega\left[U_{y}\right]\right\|_{L^{2}(e)}^{2}+h\|\omega U\|_{L^{2}(e)}^{2}\right) \\
& +\frac{h^{5 / 2}}{4 C_{i n v}^{2} C_{\omega}^{2}}\left\|\omega U_{y}\right\|_{L^{2}(\partial \Omega)}^{2}+C h^{1 / 2}\left\|\omega U n_{y}\right\|_{L^{2}(\partial \Omega)}^{2} .
\end{aligned}
$$

Since by $(2.10)$

$$
\begin{aligned}
\left\|\omega U_{y}\right\|_{L^{2}(\partial \Omega)}^{2} & =\sum_{e \in \mathcal{E}_{h}^{\partial}}\left\|\omega U_{y}\right\|_{L^{2}(e)}^{2} \leq \sum_{T \in \mathcal{T}_{h}}\left\|\omega U_{y}\right\|_{L^{2}(\partial T)}^{2} \\
& \leq C_{i n v}^{2} C_{\omega}^{2} h^{-1} \sum_{T \in \mathcal{T}_{h}}\left\|\omega U_{y}\right\|_{L^{2}(T)}^{2}=C_{i n v}^{2} C_{\omega}^{2} h^{-1}\left\|\omega U_{y}\right\|_{L^{2}(\Omega)}^{2}
\end{aligned}
$$

we obtain

$$
\begin{aligned}
h^{3 / 2} \sum_{T \in \mathcal{T}_{h}} \int_{\partial T} \omega^{2} U U_{y} n_{y} d s & \leq C h^{2} \sum_{e \in \mathcal{E}_{h}^{0}}\left\|\omega\left[U_{y}\right]\right\|_{L^{2}(e)}^{2}+C\|\omega U\|_{L^{2}(\Omega)}^{2} \\
& +\frac{h^{3 / 2}}{4}\left\|\omega U_{y}\right\|_{L^{2}(\Omega)}^{2}+C h^{1 / 2}\left\|\omega U n_{y}\right\|_{L^{2}(\partial \Omega)}^{2} .
\end{aligned}
$$

By absorbing the term $\frac{h^{3 / 2}}{4}\left\|\omega U_{y}\right\|_{L^{2}(\Omega)}^{2}$ appearing in (6.6) and (6.7) by the left hand side of (6.5), we complete the proof of the lemma.

\subsection{Proof of Lemma 3.3.}

Proof. We start the proof of the lemma by adding and subtracting the Clément interpolant of $\omega^{2} U_{x}$

$$
\left\|\omega U_{x}\right\|_{L^{2}(\Omega)}^{2}=\int_{\Omega} U_{x} \Pi\left(\omega^{2} U_{x}\right)+\int_{\Omega} U_{x}\left(\omega^{2} U_{x}-\Pi\left(\omega^{2} U_{x}\right)\right) .
$$


Using the Cauchy-Schwarz and geometric-arithmetic mean inequalities and the Lemma 3.1, we can estimate the last term on the right hand side by

$$
\begin{aligned}
\int_{\Omega} U_{x}\left(\omega^{2} U_{x}-\Pi\left(\omega^{2} U_{x}\right)\right) & \leq\left\|\omega U_{x}\right\|_{L^{2}(\Omega)}\left\|\omega^{-1}\left(\omega^{2} U_{x}-\Pi\left(\omega^{2} U_{x}\right)\right)\right\|_{L^{2}(\Omega)} \\
& \leq \frac{1}{4}\left\|\omega U_{x}\right\|_{L^{2}(\Omega)}^{2}+\left\|\omega^{-1}\left(\omega^{2} U_{x}-\Pi\left(\omega^{2} U_{x}\right)\right)\right\|_{L^{2}(\Omega)}^{2} \\
& \leq \frac{1}{4}\left\|\omega U_{x}\right\|_{L^{2}(\Omega)}^{2}+C K^{-2}\left\|\omega U_{x}\right\|_{L^{2}(\Omega)}^{2}+C h \sum_{e \in \mathcal{E}_{h}^{0}}\left\|\omega\left[U_{x}\right]\right\|_{L^{2}(e)}^{2} .
\end{aligned}
$$

By choosing $K$ large enough we can kick back the first two terms on the right hand side and from (6.8) we obtain

$$
\left\|\omega U_{x}\right\|_{L^{2}(\Omega)}^{2} \leq 2 \int_{\Omega} U_{x} \Pi\left(\omega^{2} U_{x}\right)+C h \sum_{e \in \mathcal{E}_{h}^{0}}\left\|\omega\left[U_{x}\right]\right\|_{L^{2}(e)}^{2} .
$$

Since $h^{2} \sum_{e \in \mathcal{E}_{h}^{0}}\left\|\omega\left[U_{x}\right]\right\|_{L^{2}(e)}^{2}$ is one of the terms of $Q^{2}(U)$, to complete the proof, we shall show

$$
h \int_{\Omega} U_{x} \Pi\left(\omega^{2} U_{x}\right) \leq \frac{h}{4}\left\|\omega U_{x}\right\|_{L^{2}(\Omega)}^{2}+C\left(Q^{2}(U)+L^{2}(u)\right) .
$$

To establish (6.9) we will use the orthogonality condition (1.3),

$$
B\left(U, \Pi\left(\omega^{2} U_{x}\right)\right)=B\left(u, \Pi\left(\omega^{2} U_{x}\right)\right) .
$$

Thus from (6.10),

$$
\begin{aligned}
\int_{\Omega} U_{x} \Pi\left(\omega^{2} U_{x}\right) & =B\left(u, \Pi\left(\omega^{2} U_{x}\right)\right)-\int_{\partial \Omega^{-}} U \Pi\left(\omega^{2} U_{x}\right)\left|n_{x}\right| d s-\int_{\Omega} U \Pi\left(\omega^{2} U_{x}\right) \\
& -\varepsilon A\left(U, \Pi\left(\omega^{2} U_{x}\right)\right)-J_{\|}\left(U, \Pi\left(\omega^{2} U_{x}\right)\right)-J_{\perp}\left(U, \Pi\left(\omega^{2} U_{x}\right)\right) .
\end{aligned}
$$

We bound each term of the right hand side separately. In details we will only demonstrate the estimates for $\varepsilon A\left(U, \Pi\left(\omega^{2} U_{x}\right)\right)$ and $J_{\perp}\left(U, \Pi\left(\omega^{2} U_{x}\right)\right)$. The estimates for the other terms are very similar. We start with

$$
\begin{aligned}
-\varepsilon A\left(U, \Pi\left(\omega^{2} U_{x}\right)\right)= & -\varepsilon \int_{\Omega} \nabla U \cdot \nabla \Pi\left(\omega^{2} U_{x}\right) \\
& +\varepsilon \int_{\partial \Omega}\left(\frac{\partial U}{\partial n} \Pi\left(\omega^{2} U_{x}\right)+\frac{\partial \Pi\left(\omega^{2} U_{x}\right)}{\partial n} U\right) d s \\
& -\varepsilon \gamma_{b c} h^{-1} \int_{\partial \Omega} U \Pi\left(\omega^{2} U_{x}\right) d s=I_{1}+I_{2}+I_{3} .
\end{aligned}
$$

Adding and subtracting $\omega^{2} U_{x}$ we have

$$
I_{1}=-\varepsilon \sum_{T \in \mathcal{T}_{h}} \int_{T} \nabla U \cdot \nabla\left(\omega^{2} U_{x}\right)-\varepsilon \sum_{T \in \mathcal{T}_{h}} \int_{T} \nabla U \cdot \nabla\left(\Pi\left(\omega^{2} U_{x}\right)-\omega^{2} U_{x}\right) .
$$


The first term on the right hand side we can estimate by using the properties of the weight function (2.6)-(2.8) and the fact that $U_{x}$ is piecewise constant,

$$
\begin{aligned}
\varepsilon \sum_{T \in \mathcal{T}_{h}} \int_{T} \nabla U \cdot \nabla\left(\omega^{2} U_{x}\right) & \left.=\varepsilon \sum_{T \in \mathcal{T}_{h}} \int_{T} \nabla U \cdot \nabla\left(\omega^{2}\right) U_{x}\right) \\
& =2 \varepsilon \int_{\Omega} \nabla U \cdot \nabla \omega \omega U_{x} \\
& \leq C \varepsilon K^{-1} h^{-1} \sum_{T \in \mathcal{T}_{h}}\|\omega \nabla U\|_{L^{2}(T)}^{2} \\
& =C \varepsilon K^{-1} h^{-1}\|\omega \nabla U\|_{L^{2}(\Omega)}^{2} .
\end{aligned}
$$

To estimate the other term, we use the Cauchy-Schwarz and arithmetic-geometric mean inequalities and Lemma 3.1,

$$
\begin{aligned}
& \varepsilon \sum_{T \in \mathcal{T}_{h}} \int_{T} \nabla U \cdot \nabla\left(\Pi\left(\omega^{2} U_{x}\right)-\omega^{2} U_{x}\right) \\
& \leq \frac{\varepsilon}{2} h^{-1} \sum_{T \in \mathcal{T}_{h}} \int_{T}\|\omega \nabla U\|_{L^{2}(T)}^{2}+\frac{\varepsilon}{2} h \sum_{T \in \mathcal{T}_{h}}\left\|\omega^{-1} \nabla\left(\Pi\left(\omega^{2} U_{x}\right)-\omega^{2} U_{x}\right)\right\|_{L^{2}(T)}^{2} \\
& \leq \frac{\varepsilon}{2} h^{-1}\|\omega \nabla U\|_{L^{2}(\Omega)}^{2}+C \varepsilon \sum_{e \in \mathcal{E}_{h}^{0}}\left\|\omega\left[U_{x}\right]\right\|_{L^{2}(e)}^{2}+C \varepsilon h^{-1} K^{-2}\left\|\omega U_{x}\right\|_{L^{2}(\Omega)}^{2} \\
& \leq C \varepsilon h^{-1}\|\omega \nabla U\|_{L^{2}(\Omega)}^{2}+C h \sum_{e \in \mathcal{E}_{h}^{0}}\left\|\omega\left[U_{x}\right]\right\|_{L^{2}(e)}^{2} .
\end{aligned}
$$

In the last step we used the assumption $\varepsilon \leq h$. Thus, from (6.13) and (6.14), we get

$$
\left|I_{1}\right| \leq C h^{-1} Q^{2}(U)
$$

Next, we will bound the remaining terms of $-\varepsilon A\left(U, \Pi\left(\omega^{2} U_{x}\right)\right)$, namely $I_{2}$ and $I_{3}$. By the Cauchy-Schwarz inequality

$$
\left|I_{2}\right| \leq \varepsilon\|\omega \nabla U\|_{L^{2}(\partial \Omega)}\left\|\omega^{-1} \Pi\left(\omega^{2} U_{x}\right)\right\|_{L^{2}(\partial \Omega)}+\varepsilon\left\|\omega^{-1} \nabla \Pi\left(\omega^{2} U_{x}\right)\right\|_{L^{2}(\partial \Omega)}\|\omega U\|_{L^{2}(\partial \Omega)} .
$$

By the arithmetic-geometric mean inequality

$$
\|\omega \nabla U\|_{L^{2}(\partial \Omega)}\left\|\omega^{-1} \Pi\left(\omega^{2} U_{x}\right)\right\|_{L^{2}(\partial \Omega)} \leq \frac{1}{2}\|\omega \nabla U\|_{L^{2}(\partial \Omega)}^{2}+\frac{1}{2}\left\|\omega^{-1} \Pi\left(\omega^{2} U_{x}\right)\right\|_{L^{2}(\partial \Omega)}^{2} .
$$

Using the properties of $\omega(2.8)$ and the inverse inequality (2.3),

$$
\begin{aligned}
\|\omega \nabla U\|_{L^{2}(\partial \Omega)}^{2} & \leq \sum_{e \in \mathcal{E}_{h}^{\partial}} \max _{\mathbf{x} \in e}[\omega(\mathbf{x})]^{2}\|\nabla U\|_{L^{2}(e)}^{2} \\
& \leq C_{i n v}^{2} h^{-1} \sum_{T \in \mathcal{T}_{h}} \max _{\mathbf{x} \in T}[\omega(\mathbf{x})]^{2}\|\nabla U\|_{L^{2}(T)}^{2} \\
& \leq C_{\omega}^{2} C_{i n v}^{2} h^{-1} \sum_{T \in \mathcal{T}_{h}}\|\omega \nabla U\|_{L^{2}(T)}^{2}=C_{\omega}^{2} C_{i n v}^{2} h^{-1}\|\omega \nabla U\|_{L^{2}(\Omega)}^{2} .
\end{aligned}
$$


Using (2.12), the inverse inequality (2.3), the triangle inequality, and Lemma 3.1, we have

$$
\begin{aligned}
& \left\|\omega^{-1} \Pi\left(\omega^{2} U_{x}\right)\right\|_{L^{2}(\partial \Omega)}^{2} \leq \sum_{e \in \mathcal{E}_{h}^{\partial}} \min _{\mathbf{x} \in e}[\omega(\mathbf{x})]^{-2}\left\|\Pi\left(\omega^{2} U_{x}\right)\right\|_{L^{2}(e)}^{2} \\
& \leq C_{i n v}^{2} h^{-1} \sum_{T \in \mathcal{T}_{h}} \min _{\mathbf{x} \in T}[\omega(\mathbf{x})]^{-2}\left\|\Pi\left(\omega^{2} U_{x}\right)\right\|_{L^{2}(T)}^{2} \\
& \leq C_{i n v}^{2} h^{-1} \sum_{T \in \mathcal{T}_{h}} \min _{\mathbf{x} \in T}[\omega(\mathbf{x})]^{-2}\left(\left\|\Pi\left(\omega^{2} U_{x}\right)-\omega^{2} U_{x}\right\|_{L^{2}(T)}^{2}+\left\|\omega^{2} U_{x}\right\|_{L^{2}(T)}^{2}\right) \\
& \leq C_{\omega}^{2} C_{i n v}^{2} h^{-1} \sum_{T \in \mathcal{T}_{h}}\left(\left\|\omega^{-1}\left(\Pi\left(\omega^{2} U_{x}\right)-\omega^{2} U_{x}\right)\right\|_{L^{2}(T)}^{2}+\left\|\omega U_{x}\right\|_{L^{2}(T)}^{2}\right) \\
& \leq C \sum_{e \in \mathcal{E}_{h}^{0}}\left\|\omega\left[U_{x}\right]\right\|_{L^{2}(e)}^{2}+C h^{-1} K^{-2}\left\|\omega U_{x}\right\|_{L^{2}(\Omega)}^{2}+C_{\omega}^{2} C_{i n v}^{2} h^{-1}\left\|\omega U_{x}\right\|_{L^{2}(\Omega)}^{2} \\
& \leq C \sum_{e \in \mathcal{E}_{h}^{0}}\left\|\omega\left[U_{x}\right]\right\|_{L^{2}(e)}^{2}+C h^{-1}\|\omega \nabla U\|_{L^{2}(\Omega)}^{2} .
\end{aligned}
$$

Using the assumption $\varepsilon \leq h$, we have shown,

$$
\varepsilon\|\omega \nabla U\|_{L^{2}(\partial \Omega)}\left\|\omega^{-1} \Pi\left(\omega^{2} U_{x}\right)\right\|_{L^{2}(\partial \Omega)} \leq C h^{-1} Q^{2}(U) .
$$

Similarly, by the arithmetic-geometric mean inequality

$$
\left\|\omega^{-1} \nabla \Pi\left(\omega^{2} U_{x}\right)\right\|_{L^{2}(\partial \Omega)}\|\omega U\|_{L^{2}(\partial \Omega)} \leq h^{2}\left\|\omega^{-1} \nabla \Pi\left(\omega^{2} U_{x}\right)\right\|_{L^{2}(\partial \Omega)}^{2}+(2 h)^{-2}\|\omega U\|_{L^{2}(\partial \Omega)}^{2} .
$$

Proceeding exactly as in the estimate (6.15), we obtain

$$
h^{2}\left\|\omega^{-1} \nabla \Pi\left(\omega^{2} U_{x}\right)\right\|_{L^{2}(\partial \Omega)}^{2} \leq C \sum_{e \in \mathcal{E}_{h}^{0}}\left\|\omega\left[U_{x}\right]\right\|_{L^{2}(e)}^{2}+C h^{-1}\|\omega \nabla U\|_{L^{2}(\Omega)}^{2} .
$$

Again using the assumption $\varepsilon \leq h$, we have

$$
\varepsilon\|\omega \nabla U\|_{L^{2}(\partial \Omega)}\left\|\omega^{-1} \Pi\left(\omega^{2} U_{x}\right)\right\|_{L^{2}(\partial \Omega)} \leq C h^{-1} Q^{2}(U) .
$$

Thus, from (6.16) and (6.17), we have

$$
\left|I_{2}\right| \leq C h^{-1} Q^{2}(U) .
$$

Similarly, we can show

$$
\left|I_{3}\right| \leq C h^{-1} Q^{2}(U)
$$

Thus, we have shown

$$
-\varepsilon h A\left(U, \Pi\left(\omega^{2} U_{x}\right)\right) \leq C Q^{2}(U) .
$$

In a similar fashion, we can show

$$
\begin{aligned}
& h\left|\int_{\partial \Omega^{-}} U \Pi\left(\omega^{2} U_{x}\right)\right| n_{x}|d s|+h\left|\int_{\Omega} U \Pi\left(\omega^{2} U_{x}\right)\right|+h\left|J_{\|}\left(U, \Pi\left(\omega^{2} U_{x}\right)\right)\right| \\
& +h\left|J_{\perp}\left(U, \Pi\left(\omega^{2} U_{x}\right)\right)\right| \leq C Q^{2}(U)+C h\left(K^{-2}+\delta\right)\left\|\omega U_{x}\right\|_{L^{2}(\Omega)}^{2} .
\end{aligned}
$$

We will demonstrate this for

$$
J_{\perp}\left(U, \Pi\left(\omega^{2} U_{x}\right)\right)=h^{2} \sum_{e \in \mathcal{E}_{h}^{0}} \int_{e}\left[U_{y}\right]\left[\left(\Pi\left(\omega^{2} U_{x}\right)\right)_{y}\right] d s+h^{1 / 2} \int_{\partial \Omega} U \Pi\left(\omega^{2} U_{x}\right)\left|n_{y}\right|^{2} d s .
$$


We start with the last term. By the Cauchy-Schwarz and arithmetic-geometric mean inequalities,

$$
h^{1 / 2} \int_{\partial \Omega} U \Pi\left(\omega^{2} U_{x}\right)\left|n_{y}\right|^{2} d s \leq C_{\delta} h^{-1 / 2}\left\|\omega U n_{y}\right\|_{L^{2}(\partial \Omega)}^{2}+\delta h^{3 / 2}\left\|\omega^{-1} \Pi\left(\omega^{2} U_{x}\right)\right\|_{L^{2}(\partial \Omega)}^{2}
$$

Since $h^{1 / 2}\left\|\omega U n_{y}\right\|_{L^{2}(\partial \Omega)}^{2}$ is one of the terms of $Q^{2}(U)$, we only need to treat the last term. By the properties of $\omega$, the inverse inequality (2.3), the triangle inequality, and Lemma 3.1,

$$
\begin{aligned}
& \left\|\omega^{-1} \Pi\left(\omega^{2} U_{x}\right)\right\|_{L^{2}(\partial \Omega)}^{2} \leq C_{i n v}^{2} C_{\omega}^{2} h^{-1}\left\|\omega^{-1} \Pi\left(\omega^{2} U_{x}\right)\right\|_{L^{2}(\Omega)}^{2} \\
& \leq C_{i n v}^{2} C_{\omega}^{2} h^{-1}\left(\left\|\omega^{-1}\left(\Pi\left(\omega^{2} U_{x}\right)-\omega^{2} U_{x}\right)\right\|_{L^{2}(\Omega)}^{2}+\left\|\omega U_{x}\right\|_{L^{2}(\Omega)}^{2}\right) \\
& \leq C \sum_{e \in \mathcal{E}_{h}^{0}}\left\|\omega\left[U_{x}\right]\right\|_{L^{2}(e)}^{2}+h^{-1}\left(C K^{-2}+C_{i n v}^{2} C_{\omega}^{2}\right)\left\|\omega U_{x}\right\|_{L^{2}(\Omega)}^{2} .
\end{aligned}
$$

Thus,

$$
\begin{aligned}
& h^{1 / 2} \int_{\partial \Omega} U \Pi\left(\omega^{2} U_{x}\right)\left|n_{y}\right|^{2} d s \leq C_{\delta} h^{-1 / 2}\left\|\omega U n_{y}\right\|_{L^{2}(\partial \Omega)}^{2} \\
& \quad+C h^{3 / 2} \sum_{e \in \mathcal{E}_{h}^{0}}\left\|\omega\left[U_{x}\right]\right\|_{L^{2}(e)}^{2}+\delta h^{1 / 2}\left(C K^{-2}+C_{i n v}^{2} C_{\omega}^{2}\right)\left\|\omega U_{x}\right\|_{L^{2}(\Omega)}^{2} .
\end{aligned}
$$

Next we estimate the other term of $J_{\perp}$. By the arithmetic-geometric mean inequality,

$$
h^{2} \sum_{e \in \mathcal{E}_{h}^{0}} \int_{e}\left[U_{y}\right]\left[\left(\Pi\left(\omega^{2} U_{x}\right)\right)_{y}\right] d s \leq C \sum_{e \in \mathcal{E}_{h}^{0}} h\left\|\omega\left[U_{y}\right]\right\|_{L^{2}(e)}^{2}+h^{3}\left\|\omega^{-1}\left[\left(\Pi\left(\omega^{2} U_{x}\right)\right)_{y}\right]\right\|_{L^{2}(e)}^{2} .
$$

Again, $h^{2} \sum_{e \in \mathcal{E}_{h}^{0}}\left\|\omega\left[U_{y}\right]\right\|_{L^{2}(e)}^{2}$ is one of the terms of $Q^{2}(U)$, hence we only need to treat the last term. By the properties of $\omega$, the inverse inequality (2.3), the triangle inequality, and Lemma 3.1,

$$
\begin{aligned}
h^{3} & \sum_{e \in \mathcal{E}_{h}^{0}}\left\|\omega^{-1}\left[\left(\Pi\left(\omega^{2} U_{x}\right)\right)_{y}\right]\right\|_{L^{2}(e)}^{2} \leq C h^{2} \sum_{T \in \mathcal{T}_{h}} \min _{\mathbf{x} \in T}[\omega(\mathbf{x})]^{-2}\left\|\nabla \Pi\left(\omega^{2} U_{x}\right)\right\|_{L^{2}(T)}^{2} \\
& \leq C h^{2} \sum_{T \in \mathcal{T}_{h}}\left(\left\|\omega^{-1} \nabla\left(\Pi\left(\omega^{2} U_{x}\right)-\omega^{2} U_{x}\right)\right\|_{L^{2}(T)}^{2}+\left\|\nabla \omega U_{x}\right\|_{L^{2}(T)}^{2}\right) \\
& \leq C h \sum_{e \in \mathcal{E}_{h}^{0}}\left\|\omega\left[U_{x}\right]\right\|_{L^{2}(e)}^{2}+C K^{-2}\left\|\omega U_{x}\right\|_{L^{2}(\Omega)}^{2} .
\end{aligned}
$$

Thus,

$$
\begin{aligned}
h^{2} \sum_{e \in \mathcal{E}_{h}^{0}} \int_{e}\left[U_{y}\right]\left[\left(\Pi\left(\omega^{2} U_{x}\right)\right)_{y}\right] d s & \leq C h \sum_{e \in \mathcal{E}_{h}^{0}}\left(\left\|\omega\left[U_{x}\right]\right\|_{L^{2}(e)}^{2}+\left\|\omega\left[U_{y}\right]\right\|_{L^{2}(e)}^{2}\right) \\
& +C K^{-2}\left\|\omega U_{x}\right\|_{L^{2}(\Omega)}^{2} .
\end{aligned}
$$

Combining (6.20) and (6.21) we obtain

$$
h J_{\perp}\left(U, \Pi\left(\omega^{2} U_{x}\right)\right) \leq C Q^{2}(U)+\left(C h K^{-2}+\delta h^{3 / 2} C_{i n v}^{2} C_{\omega}^{2}\right)\left\|\omega U_{x}\right\|_{L^{2}(\Omega)}^{2} .
$$

Therefore multiplying (6.11) by $h$ and using (6.18) and (6.19),

$$
h \int_{\Omega} U_{x} \Pi\left(\omega^{2} U_{x}\right) \leq C Q^{2}(U)+C h\left(K^{-2}+\delta\right)\left\|\omega U_{x}\right\|_{L^{2}(\Omega)}^{2}+h B\left(u, \Pi\left(\omega^{2} U_{x}\right)\right) .
$$


It remains to bound

$B\left(u, \Pi\left(\omega^{2} U_{x}\right)\right)=\varepsilon A\left(u, \Pi\left(\omega^{2} U_{x}\right)\right)+M\left(u, \Pi\left(\omega^{2} U_{x}\right)\right)+J_{\|}\left(u, \Pi\left(\omega^{2} U_{x}\right)\right)+J_{\perp}\left(u, \Pi\left(\omega^{2} U_{x}\right)\right)$.

The bound of the first term $\varepsilon A\left(u, \Pi\left(\omega^{2} U_{x}\right)\right.$ follows in the same fashion as (6.12).

Now, we bound

$$
M\left(u, \Pi\left(\omega^{2} U_{x}\right)\right)=\int_{\Omega} u_{x} \Pi\left(\omega^{2} U_{x}\right)+\int_{\partial \Omega^{-}} u \Pi\left(\omega^{2} U_{x}\right)\left|n_{x}\right| d s+\int_{\Omega} u \Pi\left(\omega^{2} U_{x}\right) .
$$

Adding and subtracting $\omega^{2} U_{x}$, we have

$$
\int_{\Omega} u_{x} \Pi\left(\omega^{2} U_{x}\right)=\int_{\Omega} u_{x} \omega^{2} U_{x}+\int_{\Omega} u_{x}\left(\Pi\left(\omega^{2} U_{x}\right)-\omega^{2} U_{x}\right) .
$$

By the arithmetic-geometric mean inequality

$$
\int_{\Omega} u_{x} \omega^{2} U_{x} \leq \frac{1}{16}\left\|\omega U_{x}\right\|_{L^{2}(\Omega)}^{2}+C\left\|\omega u_{x}\right\|_{L^{2}(\Omega)}^{2},
$$

and by Lemma 3.1,

$$
\begin{aligned}
\int_{\Omega} u_{x}\left(\Pi\left(\omega^{2} U_{x}\right)-\omega^{2} U_{x}\right) & \leq C\left\|\omega u_{x}\right\|_{L^{2}(\Omega)}^{2}+C\left\|\omega^{-1}\left(\Pi\left(\omega^{2} U_{x}\right)-\omega^{2} U_{x}\right)\right\|_{L^{2}(\Omega)}^{2} \\
& \leq C\left\|\omega u_{x}\right\|_{L^{2}(\Omega)}^{2}+C h^{-1} Q^{2}(U)+C K^{-2}\left\|\omega U_{x}\right\|_{L^{2}(\Omega)}^{2} .
\end{aligned}
$$

Therefore,

$h \int_{\Omega} u_{x} \Pi\left(\omega^{2} U_{x}\right) \leq \frac{h}{16}\left\|\omega U_{x}\right\|_{L^{2}(\Omega)}^{2}+C L^{2}(u)+C Q^{2}(U)+C h K^{-2}\left\|\omega U_{x}\right\|_{L^{2}(\Omega)}^{2}$.

We can easily bound the remaining terms of $M\left(u, \Pi\left(\omega^{2} U_{x}\right)\right)$ to arrive at

$$
h M\left(u, \Pi\left(\omega^{2} U_{x}\right)\right) \leq \frac{h}{8}\left\|\omega U_{x}\right\|_{L^{2}(\Omega)}^{2}+C L^{2}(u)+C Q^{2}(U)+C h K^{-2}\left\|\omega U_{x}\right\|_{L^{2}(\Omega)}^{2} .
$$

The estimates of $J_{\|}\left(u, \Pi\left(\omega^{2} U_{x}\right)\right)$ and $J_{\perp}\left(u, \Pi\left(\omega^{2} U_{x}\right)\right)$ can be derived along the lines of the estimate for $J_{\perp}\left(U, \Pi\left(\omega^{2} U_{x}\right)\right)$. Assembling all the bounds on the terms of $B\left(u, \Pi\left(\omega^{2} U_{x}\right)\right)$ we obtain

$h B\left(u, \Pi\left(\omega^{2} U_{x}\right)\right) \leq \frac{h}{6}\left\|\omega U_{x}\right\|_{L^{2}(\Omega)}^{2}+C L^{2}(u)+C Q^{2}(U)+C h\left(K^{-2}+\delta\right)\left\|\omega U_{x}\right\|_{L^{2}(\Omega)}^{2}$.

Using the above inequality, estimate (6.22), and taking $K$ large enough and $\delta$ small enough, proves (6.9). This completes the proof of the lemma.

6.4. Proof of Lemma 3.4. As we have already mentioned above, this superapproximation result is similar to the superapproximation results of [13] and [10], but here instead of a local interpolant operator we have to deal with a global $L^{2}$ projection. Because of this fact the proof is much more involved.

Proof. Recall that $E=\omega^{2} U-P\left(\omega^{2} U\right)$, where $P$ is the $L^{2}$-projection defined in (3.4). Using that $u-P u$ is orthogonal to $V_{h}$, we have

$$
\begin{aligned}
\left\|\omega^{-1} E\right\|_{L^{2}(\Omega)}^{2} & =\int_{\Omega} \omega^{-2}\left(\omega^{2} U-P\left(\omega^{2} U\right)\right)\left(\omega^{2} U-P\left(\omega^{2} U\right)\right) \\
& =\int_{\Omega}\left(\omega^{2} U-P\left(\omega^{2} U\right)\right)\left(U-\omega^{-2} P\left(\omega^{2} U\right)\right) \\
& =\int_{\Omega}\left(\omega^{2} U-P\left(\omega^{2} U\right)\right)\left(I_{h}\left(\omega^{-2} P\left(\omega^{2} U\right)\right)-\omega^{-2} P\left(\omega^{2} U\right)\right),
\end{aligned}
$$


where $I_{h}$ denotes the Lagrange interpolant.

Thus, by the Cauchy-Schwarz inequality,

$$
\left\|\omega^{-1} E\right\|_{L^{2}(\Omega)}^{2} \quad \leq\left\|\omega^{-1} E\right\|_{L^{2}(\Omega)}\left\|\omega\left(I_{h}\left(\omega^{-2} P\left(\omega^{2} U\right)\right)-\omega^{-2} P\left(\omega^{2} U\right)\right)\right\|_{L^{2}(\Omega)} .
$$

Hence,

$$
\left\|\omega^{-1} E\right\|_{L^{2}(\Omega)} \leq\left\|\omega\left(I_{h}\left(\omega^{-2} P\left(\omega^{2} U\right)\right)-\omega^{-2} P\left(\omega^{2} U\right)\right)\right\|_{L^{2}(\Omega)} .
$$

By following the proof of Lemma 2.2 in [14] and using (2.11) and (2.12), we get

$$
\begin{aligned}
& h^{-1}\left\|\omega\left(I_{h}\left(\omega^{-2} P\left(\omega^{2} U\right)\right)-\omega^{-2} P\left(\omega^{2} U\right)\right)\right\|_{L^{2}(\Omega)} \\
& \quad \leq C K^{-1 / 2} h^{-1 / 2}\left(h^{1 / 2}\left\|\omega^{-1}\left(P\left(\omega^{2} U\right)\right)_{x}\right\|_{L^{2}(\Omega)}+h^{3 / 4}\left\|\omega^{-1}\left(P\left(\omega^{2} U\right)\right)_{y}\right\|_{L^{2}(\Omega)}\right. \\
& \quad+\left\|\omega^{-1} P\left(\omega^{2} U\right)\right\|_{L^{2}(\Omega)}+\left\|\left(\omega^{-1}\left|\left(\omega^{-1}\right)_{x}\right|\right)^{1 / 2} P\left(\omega^{2} U\right)\right\|_{L^{2}(\Omega)} .
\end{aligned}
$$

Therefore by the triangle inequality,

$$
h^{-1}\left\|\omega^{-1} E\right\|_{L^{2}(\Omega)}=C K^{-1 / 2} h^{-1 / 2}\left(S_{1}+S_{2}\right),
$$

where

$$
\begin{aligned}
S_{1}= & h^{1 / 2}\left\|\omega^{-1}\left(\omega^{2} U\right)_{x}\right\|_{L^{2}(\Omega)}+h^{3 / 4}\left\|\omega^{-1}\left(\omega^{2} U\right)_{y}\right\|_{L^{2}(\Omega)} \\
& +\|\omega U\|_{L^{2}(\Omega)}+\left\|\left(\omega^{-1}\left|\left(\omega^{-1}\right)_{x}\right|\right)^{1 / 2} \omega^{2} U\right\|_{L^{2}(\Omega)} .
\end{aligned}
$$

and

$$
\begin{aligned}
S_{2} & =h^{1 / 2}\left\|\omega^{-1}\left(P\left(\omega^{2} U\right)-\omega^{2} U\right)_{x}\right\|_{L^{2}(\Omega)}+h^{3 / 4}\left\|\omega^{-1}\left(P\left(\omega^{2} U\right)-\omega^{2} U\right)_{y}\right\|_{L^{2}(\Omega)} \\
& +\left\|\omega^{-1}\left(P\left(\omega^{2} U\right)-\omega^{2} U\right)\right\|_{L^{2}(\Omega)}+\left\|\left(\omega^{-1}\left|\left(\omega^{-1}\right)_{x}\right|\right)^{1 / 2}\left(P\left(\omega^{2} U\right)-\omega^{2} U\right)\right\|_{L^{2}(\Omega)} .
\end{aligned}
$$

One can show using the product rule and (2.6), (2.7), and (2.8) that

$$
S_{1} \leq C h^{1 / 2}\left\|\omega U_{x}\right\|_{L^{2}(\Omega)}+C h^{3 / 4}\left\|\omega U_{y}\right\|_{L^{2}(\Omega)}+\|\omega U\|_{L^{2}(\Omega)}+\left\|\left(\left|\omega \omega_{x}\right|\right)^{1 / 2} U\right\|_{L^{2}(\Omega)} .
$$

Therefore, by Lemma 3.2 and Lemma 3.3, we have

$$
S_{1} \leq C(Q(U)+L(u)) .
$$

Now we bound $S_{2}$. It easily follows that

$$
\begin{aligned}
S_{2}^{2} & \leq C \sum_{T \in \mathcal{T}_{h}}\left(h\left\|\omega^{-1}\left(P\left(\omega^{2} U\right)-\omega^{2} U\right)_{x}\right\|_{L^{2}(T)}^{2}+h^{3 / 2}\left\|\omega^{-1}\left(P\left(\omega^{2} U\right)-\omega^{2} U\right)_{y}\right\|_{L^{2}(T)}^{2}\right. \\
& \left.+\left\|\omega^{-1}\left(P\left(\omega^{2} U\right)-\omega^{2} U\right)\right\|_{L^{2}(T)}^{2}+\left\|\left(\omega^{-1}\left|\left(\omega^{-1}\right)_{x}\right|\right)^{1 / 2}\left(P\left(\omega^{2} U\right)-\omega^{2} U\right)\right\|_{L^{2}(T)}^{2}\right) .
\end{aligned}
$$

We analyze the first term. By using (2.12) and the interpolation inequality (2.4), we obtain

$$
\begin{aligned}
& \sum_{T \in \mathcal{T}_{h}}\left\|\omega^{-1}\left(P\left(\omega^{2} U\right)-\omega^{2} U\right)_{x}\right\|_{L^{2}(T)}^{2}=\sum_{T \in \mathcal{T}_{h}} \min _{\mathbf{x} \in T}[\omega(\mathbf{x})]^{-2}\left\|\left(P\left(\omega^{2} U\right)-\omega^{2} U\right)_{x}\right\|_{L^{2}(T)}^{2} \\
& \leq C C_{\omega}^{2} \sum_{T \in \mathcal{T}_{h}}\left(h^{-2}\left\|\omega^{-1}\left(P\left(\omega^{2} U\right)-\omega^{2} U\right)\right\|_{L^{2}(T)}^{2}+h^{2}\left\|\omega^{-1} D^{2}\left(P\left(\omega^{2} U\right)-\omega^{2} U\right)\right\|_{L^{2}(T)}^{2}\right) .
\end{aligned}
$$

Since $P\left(\omega^{2} U\right)$ is piecewise linear,

$$
\sum_{T \in \mathcal{T}_{h}}\left\|\omega^{-1} D^{2}\left(P\left(\omega^{2} U\right)-\omega^{2} U\right)\right\|_{L^{2}(T)}^{2}=\sum_{T \in \mathcal{T}_{h}}\left\|\omega^{-1} D^{2}\left(\omega^{2} U\right)\right\|_{L^{2}(T)}^{2},
$$


and we can show using the product rule along with properties of $\omega$ that

$$
\begin{aligned}
h^{3} \sum_{T \in \mathcal{T}_{h}}\left\|\omega^{-1} D^{2}\left(\omega^{2} U\right)\right\|_{L^{2}(T)}^{2} \leq & C\left(h\left\|\omega U_{x}\right\|_{L^{2}(\Omega)}^{2}+h^{3 / 2}\left\|\omega U_{y}\right\|_{L^{2}(\Omega)}^{2}\right. \\
& \left.+\|\omega U\|_{L^{2}(\Omega)}^{2}+\left\|\left(\left|\omega \omega_{x}\right|\right)^{1 / 2} U\right\|_{L^{2}(\Omega)}^{2}\right) .
\end{aligned}
$$

Together with Lemma 3.3 and Lemma 3.2, we obtain

$$
h^{3} \sum_{T \in \mathcal{T}_{h}}\left\|\omega^{-1} D^{2}\left(P\left(\omega^{2} U\right)-\omega^{2} U\right)\right\|_{L^{2}(T)}^{2} \leq C\left(Q^{2}(U)+L^{2}(u)\right) .
$$

Therefore, we have shown

$h \sum_{T \in \mathcal{T}_{h}}\left\|\omega^{-1}\left(P\left(\omega^{2} U\right)-\omega^{2} U\right)_{x}\right\|_{L^{2}(T)}^{2} \leq C h^{-1}\left\|\omega^{-1} E\right\|_{L^{2}(\Omega)}^{2}+C\left(Q^{2}(U)+L^{2}(u)\right)$.

In a similar manner we can bound the remaining terms of $S_{2}^{2}$ to get

$$
S_{2}^{2} \leq C h^{-1}\left\|\omega^{-1} E\right\|_{L^{2}(\Omega)}^{2}+C\left(Q^{2}(U)+L^{2}(u)\right)
$$

By taking the square root of both sides we get

$$
S_{2} \leq C h^{-1 / 2}\left\|\omega^{-1} E\right\|_{L^{2}(\Omega)}+C(Q(U)+L(u)) .
$$

Therefore, if we use the bounds for $S_{1}$ and $S_{2}$, (6.23), we see that for $K$ large enough

$$
h^{-1}\left\|\omega^{-1} E\right\|_{L^{2}(\Omega)} \leq C h^{-1 / 2} K^{-1 / 2}(Q(U)+L(u)) .
$$

By (2.8) and (2.4), we get

$$
\left\|\omega^{-1} \nabla E\right\|_{L^{2}(\Omega)}^{2} \leq C h^{-2}\left\|\omega^{-1} E\right\|_{L^{2}(\Omega)}^{2}+C h^{2} \sum_{T \in \mathcal{T}_{h}}\left\|\omega^{-1} D^{2}\left(\omega^{2} U\right)\right\|_{L^{2}(\Omega)} .
$$

The proof is complete once we use the estimate (6.24).

6.5. Proof of Lemma 3.5. Presenting the proof of this lemma, we assume that the reader is already familiar with proofs of the previous lemmas. Hence, in the proof below, we skip some steps which appeared already several times in the proofs of the previous lemmas.

Proof. By adding and subtracting $B\left(U, P\left(\omega^{2} U\right)\right)$ and using the orthogonality property (1.3), we have

$$
\begin{aligned}
B\left(U, \omega^{2} U\right) & =B\left(U, \omega^{2} U-P\left(\omega^{2} U\right)\right)+B\left(U, P\left(\omega^{2} U\right)\right) \\
& =B(U, E)+B\left(u, P\left(\omega^{2} U\right)\right),
\end{aligned}
$$

with $E=\omega^{2} U-P\left(\omega^{2} U\right)$, where $P$ is the $L^{2}$-projection defined in (3.4).

First we bound $B(U, E)$. Recall that

$$
B(U, E)=\varepsilon A(U, E)+M(U, E)+J_{\|}(U, E)+J_{\perp}(U, E) .
$$

We start with

$$
\varepsilon A(U, E)=\varepsilon \int_{\Omega} \nabla U \cdot \nabla E-\varepsilon \int_{\partial \Omega}\left(\frac{\partial U}{\partial n} E+\frac{\partial E}{\partial n} U\right) d s+\frac{\gamma_{b c} \varepsilon}{h} \int_{\partial \Omega} U E d s .
$$


The first term can be bounded by using Lemma 3.4 and the assumption $\varepsilon \leq h$ as follows:

$$
\begin{aligned}
\varepsilon \int_{\Omega} \nabla U \cdot \nabla E & \leq \varepsilon\|\omega \nabla U\|_{L^{2}(\Omega)}\left\|\omega^{-1} \nabla E\right\|_{L^{2}(\Omega)} \\
& \leq C \varepsilon h^{-1 / 2} K^{-1 / 2}\|\omega \nabla U\|_{L^{2}(\Omega)}(Q(U)+L(u)) \\
& \leq C K^{-1 / 2} \varepsilon\|\omega \nabla U\|_{L^{2}(\Omega)}^{2}+C K^{-1 / 2}\left(Q^{2}(U)+L^{2}(u)\right) \\
& \leq C K^{-1 / 2}\left(Q^{2}(U)+L^{2}(u)\right)
\end{aligned}
$$

The remaining terms of $\varepsilon A(U, E)$ can be bounded in a similar way. Thus we get

$$
\varepsilon A(U, E) \leq C K^{-1 / 2}\left(Q^{2}(U)+L^{2}(u)\right) .
$$

The next term we will treat is

$$
M(U, E)=\int_{\Omega} U_{x} E+\int_{\partial \Omega^{-}} U E\left|n_{x}\right| d s+\int_{\Omega} U E .
$$

By using that $E$ is orthogonal to $V_{h}$, the Cauchy-Schwarz inequality, Lemma 3.1 and Lemma 3.4,

$$
\begin{aligned}
\int_{\Omega} U_{x} E= & \int_{\Omega}\left(U_{x}-\Pi\left(U_{x}\right)\right) E \\
& \leq C\left\|h^{1 / 2} \omega\left(U_{x}-\Pi\left(U_{x}\right)\right)\right\|_{L^{2}(\Omega)} h^{-1 / 2}\left\|\omega^{-1} E\right\|_{L^{2}(\Omega)} \\
& \leq C \sum_{e \in \mathcal{E}_{h}^{0}} h^{2}\left\|\omega\left[U_{x}\right]\right\|_{L^{2}(e)}^{2}+h^{-1}\left\|\omega^{-1} E\right\|_{L^{2}(\Omega)}^{2} \\
& \leq C K^{-1} Q^{2}(U)+C L^{2}(u) .
\end{aligned}
$$

Similarly, we can bound the last two terms of $M(U, E)$. Thus, we obtain

$$
M(U, E) \leq C K^{-1 / 2} Q^{2}(U)+C L^{2}(u) .
$$

In a similar fashion we may bound the remaining terms of $B(U, E)$ (following the proof of Lemma 3.3) to get

$$
B(U, E) \leq C K^{-1 / 2} Q^{2}(U)+C L^{2}(u) .
$$

It remains to estimate $B\left(u, P\left(\omega^{2} U\right)\right)$.

We start with

$$
\begin{aligned}
\varepsilon A\left(u, P\left(\omega^{2} U\right)\right)= & \varepsilon \int_{\Omega} \nabla u \cdot \nabla P\left(\omega^{2} U\right)-\varepsilon \int_{\partial \Omega}\left(\frac{\partial u}{\partial n} P\left(\omega^{2} U\right)+\frac{\partial P\left(\omega^{2} U\right)}{\partial n} u\right) d s \\
& +\varepsilon \gamma_{b c} h^{-1} \int_{\partial \Omega} u P\left(\omega^{2} U\right) d s .
\end{aligned}
$$

Using the Cauchy-Schwarz, arithmetic-geometric mean, and the triangle inequalities, we can bound the first term on the right hand side as follows:

$$
\begin{aligned}
& \varepsilon \int_{\Omega} \nabla u \cdot \nabla P\left(\omega^{2} U\right) \leq C \varepsilon\|\omega \nabla u\|_{L^{2}(\Omega)}\left\|\omega^{-1} \nabla P\left(\omega^{2} U\right)\right\|_{L^{2}(\Omega)} \\
& \leq C_{\delta} \varepsilon\|\omega \nabla u\|_{L^{2}(\Omega)}^{2}+\varepsilon \delta\left(\left\|\omega^{-1} \nabla E\right\|_{L^{2}(\Omega)}^{2}+\left\|\omega^{-1} \nabla\left(\omega^{2} U\right)\right\|_{L^{2}(\Omega)}^{2}\right),
\end{aligned}
$$

where $\delta$ is some small number to be chosen later.

By the superapproximation result, Lemma 3.4, we have

$$
\left\|\omega^{-1} \nabla E\right\|_{L^{2}(\Omega)}^{2} \leq C h^{-1} K^{-1}\left(Q^{2}(U)+L^{2}(u)\right),
$$


and by the triangle inequality

$$
\left\|\omega^{-1} \nabla\left(\omega^{2} U\right)\right\|_{L^{2}(\Omega)}^{2} \leq\|\omega \nabla U\|_{L^{2}(\Omega)}^{2}+4\|\nabla \omega U\|_{L^{2}(\Omega)}^{2} .
$$

By the properties of the weight function (2.6) and (2.7), we have

$$
\|\nabla \omega U\|_{L^{2}(\Omega)}^{2}=\int_{\Omega} \omega_{x}^{2} U^{2}+\int_{\Omega} \omega_{y}^{2} U^{2} \leq C h^{-1} K^{-1} \int_{\Omega} \omega\left|\omega_{x}\right| U^{2}+C \sigma^{-2} K^{-2} \int_{\Omega} \omega^{2} U^{2} .
$$

Using that $\varepsilon \leq h$ and $\varepsilon \leq \sigma^{2}$ we have

$$
\begin{aligned}
& \varepsilon\left(\nabla u, \nabla P\left(\omega^{2} U\right)\right) \leq C_{\delta} \varepsilon\|\omega \nabla u\|_{L^{2}(\Omega)}^{2}+C \delta K^{-1}\left(Q^{2}(U)+L^{2}(u)\right) \\
& +C \delta K^{-1}\left\|\left(\omega\left|\omega_{x}\right|\right)^{1 / 2} U\right\|_{L^{2}(\Omega)}^{2}+C \delta K^{-2}\|\omega U\|_{L^{2}(\Omega)}^{2}+C \delta \varepsilon\|\omega \nabla U\|_{L^{2}(\Omega)}^{2} \\
& \leq C_{\delta} \varepsilon\|\omega \nabla u\|_{L^{2}(\Omega)}^{2}+C \delta K^{-1}\left(Q^{2}(U)+L^{2}(u)\right) .
\end{aligned}
$$

Now we will treat the $\varepsilon \gamma_{b c} h^{-1} \int_{\partial \Omega} u P\left(\omega^{2} U\right) d s$ term. By the arithmetic-geometric mean and triangle inequalities, we have

$$
\int_{\partial \Omega} u P\left(\omega^{2} U\right) d s \leq C_{\delta}\|\omega u\|_{L^{2}(\partial \Omega)}^{2}+\delta\left\|\omega^{-1} E\right\|_{L^{2}(\partial \Omega)}^{2}+\delta\|\omega U\|_{L^{2}(\partial \Omega)}^{2} .
$$

By the trace inequality $(2.1)$

$$
\begin{aligned}
\|\omega u\|_{L^{2}(\partial \Omega)}^{2} & \leq C h^{-1}\|\omega u\|_{L^{2}(\Omega)}^{2}+C h\|\nabla(\omega u)\|_{L^{2}(\Omega)}^{2} \\
& \leq C h^{-1}\|\omega u\|_{L^{2}(\Omega)}^{2}+C h\left(\|\nabla \omega u\|_{L^{2}(\Omega)}^{2}+\|\omega \nabla u\|_{L^{2}(\Omega)}^{2}\right) .
\end{aligned}
$$

Using (2.7), we have

$$
\|\nabla \omega u\|_{L^{2}(\Omega)}^{2} \leq C h^{-2} K^{-2}\|\omega u\|_{L^{2}(\Omega)}^{2} .
$$

Thus,

$$
\|\omega u\|_{L^{2}(\partial \Omega)}^{2} \leq C\left(h^{-1}\|\omega u\|_{L^{2}(\Omega)}^{2}+h\|\omega \nabla u\|_{L^{2}(\Omega)}^{2}\right) \leq C L^{2}(u) .
$$

By the Remark 2,

$$
\left\|\omega^{-1} E\right\|_{L^{2}(\partial \Omega)}^{2} \leq C K^{-1}\left(Q^{2}(U)+L^{2}(u)\right) .
$$

Thus,

$$
\varepsilon \gamma_{b c} h^{-1} \int_{\partial \Omega} u P\left(\omega^{2} U\right) d s \leq C_{\delta} L^{2}(u)+C K^{-1} Q^{2}(U)+C \delta Q^{2}(U) .
$$

Next we bound

$$
J_{\|}\left(u, P\left(\omega^{2} U\right)\right)=h^{2} \sum_{e \in \mathcal{E}_{h}^{0}} \int_{e}\left[u_{x}\right]\left[\left(P\left(\omega^{2} U\right)\right)_{x}\right] d s .
$$

By the Cauchy-Schwarz and the triangle inequalities

$$
h^{2} \int_{e}\left[u_{x}\right]\left[\left(P\left(\omega^{2} U\right)\right)_{x}\right] d s \leq C_{\delta} h^{2}\|\omega[\nabla u]\|_{L^{2}(e)}^{2}+\delta h^{2}\left\|\omega^{-1}\left[\left(P\left(\omega^{2} U\right)\right)_{x}\right]\right\|_{L^{2}(e)}^{2} .
$$

By (2.12), the inverse inequality (2.3), and the triangle inequality, we have

$$
\begin{aligned}
\sum_{e \in \mathcal{E}_{h}^{0}} h^{2}\left\|\omega^{-1}\left[\left(P\left(\omega^{2} U\right)\right)_{x}\right]\right\|_{L^{2}(e)}^{2} & \leq C \sum_{T \in \mathcal{T}_{h}} h\left\|\omega^{-1}\left(P\left(\omega^{2} U\right)\right)_{x}\right\|_{L^{2}(T)}^{2} \\
& \leq C h\left(\left\|\omega^{-1}\left(\omega^{2} U\right)_{x}\right\|_{L^{2}(\Omega)}^{2}+\left\|\omega^{-1} E_{x}\right\|_{L^{2}(\Omega)}^{2}\right) \\
& \leq C h\left(\left\|\omega_{x} U\right\|_{L^{2}(\Omega)}^{2}+\left\|\omega U_{x}\right\|_{L^{2}(\Omega)}^{2}+\left\|\omega^{-1} E_{x}\right\|_{L^{2}(\Omega)}^{2}\right)
\end{aligned}
$$


Since

$$
h\left\|\omega_{x} U\right\|_{L^{2}(\Omega)}^{2} \leq C K^{-1}\left\|\left(\omega\left|\omega_{x}\right|\right)^{1 / 2} U\right\|_{L^{2}(\Omega)}^{2},
$$

by Lemma 3.3 and Lemma 3.4, we have

$$
\sum_{e \in \mathcal{E}_{h}^{0}} h^{2}\left\|\omega^{-1}\left[\left(P\left(\omega^{2} U\right)\right)_{x}\right]\right\|_{L^{2}(e)}^{2} \leq C\left(K^{-1} Q^{2}(U)+Q^{2}(U)+L^{2}(u)\right) .
$$

Hence

$$
J_{\|}\left(u, P\left(\omega^{2} U\right)\right) \leq C\left(\delta Q^{2}(U)+L^{2}(u)\right) .
$$

The estimate of $J_{\perp}\left(u, P\left(\omega^{2} U\right)\right)$ is similar. It remains to bound

$$
M\left(u, P\left(\omega^{2} U\right)\right)=-M(u, E)+M\left(u, \omega^{2} U\right)
$$

The first term can be controlled by using the Cauchy-Schwarz inequality and the superapproximation result of Lemma 3.4. The second term we integrate by parts and split the term in the following fashion

$$
\begin{aligned}
\left|M\left(u, \omega^{2} U\right)\right| & =\int_{\Omega}\left(u \omega_{x} \omega U+u \omega^{2} U_{x}\right) \\
& \leq h^{-\frac{1}{2}}\|\omega u\|_{L^{2}(\Omega)}\left\|\left(\omega\left|\omega_{x}\right|\right)^{\frac{1}{2}} U\right\|_{L^{2}(\Omega)}+h^{-\frac{1}{2}}\|\omega u\|_{L^{2}(\Omega)} h^{\frac{1}{2}}\left\|\omega U_{x}\right\|_{L^{2}(\Omega)} .
\end{aligned}
$$

Similarly to the analysis above, we obtain,

$$
\left|M\left(u, \omega^{2} U\right)\right| \leq \delta\left(Q^{2}(U)+h\left\|\omega U_{x}\right\|_{L^{2}(\Omega)}^{2}\right)+C_{\delta} L^{2}(u) .
$$

Thus,

$$
B\left(u, P\left(\omega^{2} U\right)\right) \leq C K^{-1} Q^{2}(U)+C \delta\left(Q^{2}(U)+h\left\|\omega U_{x}\right\|_{L^{2}(\Omega)}^{2}\right)+C_{\delta} L^{2}(u) .
$$

Combining estimates (6.28) and (6.29) we conclude the proof of the lemma.

Acknowledgments: The authors would like to thank the anonymous reviewers for very insightful comments and for helping to improve the presentation of the paper.

\section{REFERENCES}

[1] R. Becker and M. Braack, Two-level stabilization scheme for the Navier-Stokes equations, Numerical Mathematics and Advanced Applications, 123-130, Springer, Berlin, 2004.

[2] E. Burman and P. Hansbo, Edge stabilization for Galerkin approximations of convectiondiffusion-reaction problems, Comput. Methods Appl. Mech. Engrg. 193 (2004), 1437-1453.

[3] E. Burman, A unified analysis of conforming and non-conforming stabilized finite element methods using interior penalty, SIAM J. Num. Anal. 43 (2005), 2012-2032.

[4] E. Burman, M. Fernández, and P. Hansbo, Continuous Interior Penalty for Oseen's Equations, SIAM J. Num. Anal. 44 (2006), 1248-1274.

[5] E. Burman and A. Ern, Continuous interior penalty hp-finite element methods for advection and advection-diffusion equations, Math. Comp. 76 (2007), 1119-1140.

[6] P.G. Ciarlet, The Finite Element Method for Elliptic Problems, North-Holland, Amsterdam, 1978.

[7] P. Clément, Approximation by finite elements functions using local regularization, RAIRO Anal. Numer. 9 (1975), 77-84.

[8] R. Codina and J. Blasco, Analysis of a stabilized finite element approximation of the transient convection-diffusion-reaction equation using orthogonal subscales. Comput. Vis. Sci. 4 (2002), no. $3,167-174$.

[9] J. Douglas and T. Dupont, Interior penalty procedures for elliptic and parabolic Galerkin methods In Computing methods in applied sciences (Second Internat. Sympos., Versailles, 1975), pages 207-216. Lecture Notes in Phys., Vol. 58. Springer, Berlin, (1976).

[10] P. Grisvard, Singularities in Boundary Value Problems, Recherches en Mathmatiques Appliques [Research in Applied Mathematics], 22. Masson, Paris; Springer-Verlag, Berlin, 1992. 
[11] J.-L. Guermond. Stabilization of Galerkin approximations of transport equations by subgrid modeling. M2AN Math. Model. Numer. Anal. 33 (1999), no. 6, 1293-1316.

[12] J. Guzmán, Local analysis of discontinuous Galerkin methods applied to singularly perturbed problems, J. Numer. Math. 14 (2006), 41-56.

[13] C. Johnson, U. Nävert, J. Pitkänta, Finite element methods for linear hyperbolic problems, Comput. Methods Appl. Mech. Engrg. 45 (1984), 285-312.

[14] C. Johnson, A.H. Schatz, and L.B. Wahlbin, Crosswind smear and pointwise errors in streamline diffusion finite element methods, Math. Comp. 49 (1987), 25-38.

[15] K. Niijima, Pointwise error estimates for a streamline diffusion finite element scheme, Numer. Math. 56 (1990), 707-719.

[16] G. Sangalli, Global and local error analysis for the residual-free bubbles method applied to advection-dominated problems, SIAM J. Numer. Anal. 38 (2000), 1496-1522.

Department of Mathematics, University of Sussex, Brighton, BN1 9RF UK, email: E.N.Burman@sussex.ac.uk.

School of Mathematics, University of Minnesota, Minneapolis, Mn 55455, USA, email: guzma033@umn.edu.

Department of Mathematics, University of Connecticut, Storrs, CT 06269, USA, EMAIL: leykekhman@math.uconn.edu. 